\title{
On Exponential Stability for a Class of Uncertain Neutral Markovian Jump Systems with Mode-Dependent Delays
}

\author{
Xinghua Liu and Hongsheng Xi \\ Department of Auto, School of Information Science and Technology, University of Science and Technology of China, \\ Anhui 230027, China \\ Correspondence should be addressed to Xinghua Liu; salxh@mail.ustc.edu.cn \\ Received 10 May 2013; Revised 10 July 2013; Accepted 10 July 2013 \\ Academic Editor: Elena Braverman \\ Copyright (C) 2013 X. Liu and H. Xi. This is an open access article distributed under the Creative Commons Attribution License, \\ which permits unrestricted use, distribution, and reproduction in any medium, provided the original work is properly cited. \\ The exponential stability of neutral Markovian jump systems with interval mode-dependent time-varying delays, nonlinear \\ perturbations, and partially known transition rates is investigated. A novel augmented stochastic Lyapunov functional is \\ constructed, which employs the improved bounding technique and contains triple-integral terms to reduce conservativeness; \\ then the delay-range-dependent and rate-dependent exponential stability criteria are developed by Lyapunov stability theory, \\ reciprocally convex lemma, and free-weighting matrices. The corresponding results are extended to the uncertain case. Finally, \\ numerical examples are given to illustrate the effectiveness of the proposed methods.
}

\section{Introduction}

Delay differential equations or systems are assuming an increasingly important role in many disciplines like mathematics, science, and engineering. In particular, the stability and stabilization problem for neutral delay differential dynamic systems have received considerable attention during the decades and neutral time-delay systems have been the focus of the research community, which are often encountered in such practical situations as distributed networks, population ecology, processes including steam or heat exchanges [1], and robots in contact with rigid environments [2]. Existing results can be roughly classified into two categories, delay-independent criteria and delay-dependent criteria, where the latter is generally regarded as less conservative. Moreover, since the derivative of the delayed state is involved, it should be pointed out that the stability of neutral time-delay systems is more difficult to tackle, which is identical with singular systems $[3,4]$. The stability problem of them is more complicated than that for regular systems because more factors need to be considered. In the past decades, considerable attention has been devoted to the robust delay-independent stability and delay-dependent stability of linear neutral systems, which are mainly obtained based on the LyapunovKrasovskii (L-K) method [5-11], and references therein. It should be noted that the delay-partitioning approach is used in [6-8]. Furthermore, when nonlinear perturbations or parameter uncertainties appear in neutral systems, some results on stability analysis have been also presented [12-18]. Various techniques have been proposed in these papers, for example, model transformation techniques, the improved bounding techniques, and matrix decomposition approaches. In particular, $\mathrm{He}$ et al. [18] propose a new method for dealing with time-delay systems, which employs free weighting matrices to express the relationships between the terms in the Newton-Leibniz formula and has brought novel results. However, these results have conservativeness to some extent, which exist room for further improvement.

In another line, Markovian jump systems (MJSs) have attracted much attention during the past few decades since its first introduction by Krasovskii and Lidskii in 1961, which can be regarded as a special class of hybrid systems with finite operation modes whose structures are subject to random abrupt changes. The system parameters usually jump among finite modes, and the mode switching is governed by a Markov process. MJSs have many applications, such as failure 
prone manufacturing systems, power systems, solar thermal central receivers, robotic manipulator systems, aircraft control systems, and economic systems. A large number of results on estimation and control problems related to such systems have been reported in the literature; see, for example, [19-25] and references therein for more details. However, these lines of literature about the transition probabilities in the jumping process have been assumed to be completely accessible. The ideal assumption on the transition probabilities inevitably limits the application of the traditional Markovian jump system theory. Actually, the likelihood of obtaining such available knowledge is questionable, and the cost may be very expensive. Thus, it is really significant and meaningful, from control perspectives, to further study more general jump systems with partially known transition rates. Recently, many results on the Markovian jump systems with partially known transition rates are obtained [26-31]. Most of these improved results just require some free matrices or the knowledge of the known elements in transition rate matrix, such as the structures of uncertainties, and some else of the unknown elements need not be considered. It is a great progress on the analysis of Markovian jump systems. However, few of these results are concerned with neutral Markovian jump systems with mode-dependent time-varying delays and perturbations. To the best of the authors' knowledge, neutral Markovian jump systems with mode-dependent timevarying delays and partially known transition rates have not been fully investigated, and it is very challenging, especially when nonlinear perturbations exist. Besides, seeking and proposing less conservative delay-range-dependent criterion for uncertain neutral MJSs with nonlinear perturbations and partially known transition rates to desired performance are still open problems. These facts thus motivate our study.

In this paper, the investigated neutral Markovian jump systems are more general than the neutral MJSs with completely known or completely unknown transition rates, which can be viewed as two special cases of the ones tackled here. Specifically, a new augmented stochastic Lyapunov functional containing triple-integral terms is constructed by dividing the delay interval into two subintervals, and then the delayrange-dependent and rate-dependent exponential stability criteria are obtained by reciprocally convex lemma and free weighting matrices. We further extend the criteria to the uncertain case. All the obtained results are presented in terms of LMIs that can be solved numerically. The remainder of the paper is organized as follows. Section 2 presents the problem and preliminaries. Section 3 gives the main results, which are then verified by numerical examples in Section 4 . Section 5 concludes the paper.

Notations. The following notations are used throughout the paper. $\mathbb{R}^{n}$ denotes the $n$ dimensional Euclidean space and $\mathbb{R}^{m \times n}$ is the set of all $m \times n$ matrices. $X<Y(X>Y)$, where $X$ and $Y$ are both symmetric matrices, means that $X-Y$ is negative (positive) definite. $I$ is the identity matrix with proper dimensions. For a symmetric block matrix, we use * to denote the terms introduced by symmetry. $\mathscr{E}$ stands for the mathematical expectation, $\|v\|$ is the Euclidean norm of vector $v,\|v\|=\left(v^{T} v\right)^{1 / 2}$, while $\|A\|$ is spectral norm of matrix
$A,\|A\|=\left[\lambda_{\max }\left(A^{T} A\right)\right]^{1 / 2} \cdot \lambda_{\max (\min )}(A)$ is the eigenvalue of matrix $A$ with maximum(minimum) real part. Matrices, if their dimensions are not explicitly stated, are assumed to have compatible dimensions for algebraic operations.

\section{Problem Statement and Preliminaries}

Given a probability space $\{\Omega, \mathscr{F}, \mathbf{P}\}$ where $\Omega$ is the sample space, $\mathscr{F}$ is the algebra of events and $\mathbf{P}$ is the probability measure defined on $\mathscr{F}$. $\left\{r_{t}, t \geq 0\right\}$ is a homogeneous, finitestate Markovian process with right continuous trajectories taking values in a finite set $S=\{1,2,3, \ldots, N\}$, with the mode transition probability matrix being

$$
P\left(r_{t+\Delta t}=j \mid r_{t}=i\right)= \begin{cases}\pi_{i j} \Delta t+o(\Delta t), & i \neq j, \\ 1+\pi_{i i} \Delta t+o(\Delta t), & i=j,\end{cases}
$$

where $\Delta t>0, \lim _{\Delta t \rightarrow 0}(o(\Delta t) / \Delta t)=0, \pi_{i j} \geq 0(i, j \in S, i \neq j)$ is the transition rate from mode $i$ to $j$ and for any state or mode $i \in S$; it satisfies

$$
\pi_{i i}=-\sum_{j=1, j \neq i}^{N} \pi_{i j}
$$

Since the transition rates of the Markov chain are partially known in this paper, some elements in matrix $\Pi=\left[\pi_{i j}\right]_{N \times N}$ are inaccessible. For instance, the system with five operation modes, the jump rates matrix $\Pi$ may be viewed as

$$
\left[\begin{array}{ccccc}
? & \pi_{12} & ? & ? & \pi_{15} \\
\pi_{21} & ? & ? & \pi_{24} & ? \\
? & ? & \pi_{33} & \pi_{34} & ? \\
? & \pi_{42} & ? & ? & ? \\
\pi_{51} & ? & ? & ? & \pi_{55}
\end{array}\right]
$$

where? represents the unknown element. For notation clarity, we denote $\mathcal{S}^{i}=\mathcal{S}_{k}^{i} \cup \mathcal{S}_{u k}^{i}$, for all $i \in S$ and

$$
\begin{gathered}
\mathcal{S}_{k}^{i} \triangleq\left\{j: \pi_{i j} \text { is known for } j \in S\right\}, \\
\mathcal{S}_{u k}^{i} \triangleq\left\{j: \pi_{i j} \text { is unknown for } j \in S\right\} .
\end{gathered}
$$

If $\delta_{k}^{i} \neq \emptyset$, it is further described as

$$
\mathcal{S}_{k}^{i}=\left\{k_{1}^{i}, k_{2}^{i}, \ldots, k_{m}^{i}\right\}, \quad 1 \leq m \leq N
$$

where $k_{j}^{i},(j=1,2, \ldots, m)$ represent the $j$ th known element of the set $\delta_{k}^{i}$ in the $i$ th row of the transition rate matrix $\Pi$. Furthermore, let $\pi_{i}^{m}$ and $\pi_{i}^{M}$ be the lower and upper bound for the diagonal elements of the jump rates matrix $\Pi$.

In this paper, the following uncertain neutral Markovian jump systems with mode-dependent interval time-varying 
delays, nonlinear perturbations, and partially known transition rates over the space $\{\Omega, \mathscr{F}, \mathbf{P}\}$ are considered:

$$
\begin{aligned}
\dot{x}(t)- & C\left(t, r_{t}\right) \dot{x}\left(t-\tau\left(t, r_{t}\right)\right) \\
= & A\left(t, r_{t}\right) x(t)+B\left(t, r_{t}\right) x\left(t-d\left(t, r_{t}\right)\right) \\
& +D\left(t, r_{t}\right) f_{1}(x(t), t) \\
& +E\left(t, r_{t}\right) f_{2}\left(x\left(t-d\left(t, r_{t}\right)\right), t\right) \\
& +F\left(t, r_{t}\right) f_{3}\left(\dot{x}\left(t-\tau\left(t, r_{t}\right)\right), t\right), \\
x(s) & =\varphi(s), \quad r_{s}=r_{0}, \quad s \in[-\varsigma, 0],
\end{aligned}
$$

where $x(t) \in \mathbb{R}^{n}$ is the system state and $\tau\left(t, r_{t}\right)$ is modedependent interval time-varying neutral delay which satisfies $0 \leq \tau_{i}(t) \leq \bar{\tau}_{i}, \dot{\tau}_{i}(t) \leq v_{i}$ when $r_{t}=i \in S$. The mode-dependent interval time-varying retarded delay $d\left(t, r_{t}\right)$ is assumed that

$$
\begin{gathered}
0 \leq d_{1 i} \leq d_{i}(t) \leq d_{2 i}, \quad \max _{i \in S}\left\{d_{1 i}\right\} \leq \min _{j \in S}\left\{d_{2 j}\right\}, \\
\dot{d}_{i}(t) \leq \mu_{i}, \quad \text { if } r_{t}=i \in S,
\end{gathered}
$$

where $\overline{\tau_{i}}, v_{i} \geq 0, d_{1 i}, d_{2 i}, \mu_{i} \geq 0$, and $\varsigma=\max _{i \in S}\left\{\overline{\tau_{i}}, d_{2 i}\right\}$ are real constant scalars. The initial condition $\varphi(s)$ is a continuously differentiable vector-valued function. $f_{1}(x(t), t) \in \mathbb{R}^{n}$, $f_{2}\left(x\left(t-d\left(t, r_{t}\right)\right), t\right) \in \mathbb{R}^{n}$, and $f_{3}\left(\dot{x}\left(t-\tau\left(t, r_{t}\right)\right), t\right) \in \mathbb{R}^{n}$ are unknown nonlinear perturbations which are, with respect to the current state $x(t)$, the delayed state $x\left(t-d\left(t, r_{t}\right)\right)$ and the neutral delay state $\dot{x}\left(t-\tau\left(t, r_{t}\right)\right)$, respectively. For all $t$ and $r_{t}=i \in S$, they are assumed to be bounded in magnitude as

$$
\begin{aligned}
\left\|f_{1}(x(t), t)\right\| & \leq \alpha\|x(t)\|, \\
\left\|f_{2}\left(x\left(t-d_{i}(t)\right), t\right)\right\| & \leq \beta\left\|x\left(t-d_{i}(t)\right)\right\|, \\
\left\|f_{3}\left(\dot{x}\left(t-\tau_{i}(t)\right), t\right)\right\| & \leq \gamma\left\|\dot{x}\left(t-\tau_{i}(t)\right)\right\|,
\end{aligned}
$$

where $\alpha \geq 0, \beta \geq 0$, and $\gamma \geq 0$ are given constants, for simplicity, $f_{1} \triangleq f_{1}(x(t), t), f_{2} \triangleq f_{2}\left(x\left(t-d_{i}(t)\right), t\right)$, and $f_{3} \triangleq$ $f_{3}\left(\dot{x}\left(t-\tau_{i}(t)\right), t\right)$.

For notational simplicity further, where $r_{t}=i \in S$, the parametric matrices $A\left(t, r_{t}\right) \in \mathbb{R}^{n \times n}, B\left(t, r_{t}\right) \in \mathbb{R}^{n \times n}, C\left(t, r_{t}\right) \in$ $\mathbb{R}^{n \times n}, D\left(t, r_{t}\right) \in \mathbb{R}^{n \times n}, E\left(t, r_{t}\right) \in \mathbb{R}^{n \times n}$, and $F\left(t, r_{t}\right) \in \mathbb{R}^{n \times n}$ are denoted by $A_{i}(t), B_{i}(t), C_{i}(t), D_{i}(t), E_{i}(t)$, and $F_{i}(t)$, which can be described as

$$
\begin{gathered}
A_{i}(t)=A_{i}+\Delta A_{i}(t), \quad B_{i}(t)=B_{i}+\Delta B_{i}(t), \\
C_{i}(t)=C_{i}, \quad D_{i}(t)=D_{i}+\Delta D_{i}(t), \\
E_{i}(t)=E_{i}+\Delta E_{i}(t), \quad F_{i}(t)=F_{i}+\Delta F_{i}(t),
\end{gathered}
$$

where $A_{i}, B_{i}, C_{i}, D_{i}, E_{i}$, and $F_{i}$ are known constant matrices with appropriate dimensions. $\Delta A_{i}(t) \in \mathbb{R}^{n \times n}, \Delta B_{i}(t) \in \mathbb{R}^{n \times n}$, $\Delta D_{i}(t) \in \mathbb{R}^{n \times n}, \Delta E_{i}(t) \in \mathbb{R}^{n \times n}$, and $\Delta F_{i}(t) \in \mathbb{R}^{n \times n}$ are uncertainties. The parametric matrix $\left\|C_{i}\right\|<1$ and the admissible parametric uncertainties satisfy the following condition:

$$
\begin{array}{r}
{\left[\begin{array}{lllll}
\Delta A_{i}(t) & \Delta B_{i}(t) & \Delta D_{i}(t) & \Delta E_{i}(t) & \Delta F_{i}(t)
\end{array}\right]} \\
=L_{i} H_{i}(t)\left[\begin{array}{lllll}
N_{A i} & N_{B i} & N_{D i} & N_{E i} & N_{F i}
\end{array}\right]
\end{array}
$$

where $L_{i}, N_{A i}, N_{B i}, N_{D i}, N_{E i}$, and $N_{F i}$ are known modedependent constant matrices with appropriate dimensions and $H_{i}(t)$ is an unknown and time-varying matrix satisfying

$$
H_{i}^{T}(t) H_{i}(t) \leq I, \quad \forall t .
$$

Particularly, the following nominal systems can be obtained for $H_{i}(t)=0$ :

$$
\begin{aligned}
\dot{x}(t)-C_{i} \dot{x}\left(t-\tau_{i}(t)\right)= & A_{i} x(t)+B_{i} x\left(t-d_{i}(t)\right) \\
& +D_{i} f_{1}+E_{i} f_{2}+F_{i} f_{3} .
\end{aligned}
$$

Before proceeding with the main results, we present the following assumptions, definitions, and lemmas.

Assumption 1. System matrices $A_{i}$, (for all $i \in S$ ), are Hurwitz and all the eigenvalues have negative real parts for each mode. $L_{i}$, (for all $i \in S$ ), is full rank in row.

Assumption 2. The Markov process is irreducible and the system mode $r_{t}$ is available at time $t$.

Definition 3 (see [32]). Define operator $\mathfrak{D}: C\left([-\varsigma, 0], \mathbb{R}^{n}\right) \rightarrow$ $\mathbb{R}^{n}$ as $\mathfrak{D}\left(x_{t}\right)=x(t)-C x(t-\tau)$. $\mathfrak{D}$ is said to be stable if the homogeneous difference equation

$$
\begin{gathered}
\mathfrak{D}\left(x_{t}\right)=0, \quad t \geq 0, \\
x_{0}=\psi \in\left\{\phi \in C\left([-\varsigma, 0], \mathbb{R}^{n}\right): \mathfrak{D} \phi=0\right\}
\end{gathered}
$$

is uniformly asymptotically stable. In this paper, that is, $\left\|C_{i}\right\|+$ $\gamma<1$.

Definition 4 (see [33]). The system in (6) is exponentially stable with a decay rate $\varepsilon$ for all $r_{t}=i \in S$, if there exist scalars $\varepsilon>0$ and $\kappa \geq 1$ such that for all $x(t)$,

$$
\|x(t)\| \leq \kappa \exp \left\{-\varepsilon\left(t-t_{0}\right)\right\}\left\|x_{t_{0}}\right\|_{\zeta},
$$

where $\varepsilon$ is the exponential decay rate, $\|*\|$ denotes the Euclidean norm, and

$$
\begin{aligned}
\left\|x_{t_{0}}\right\|_{\varsigma} & =\sup _{\theta \in[-\varsigma, 0]}\left\{\left\|x\left(t_{0}+\theta\right)\right\|,\left\|\dot{x}\left(t_{0}+\theta\right)\right\|\right\} \\
& =\sup _{s \in\left[-\varsigma, t_{0}\right]}\{\|\varphi(s)\|,\|\dot{\varphi}(s)\|\} .
\end{aligned}
$$

Definition 5 (see [34]). Define the stochastic LyapunovKrasovskii function of system (6) as $V\left(x(t), r_{t}=i, t>0\right)=$ $V\left(x_{t}, i, t\right)$, where its infinitesimal generator is defined as

$$
\begin{aligned}
& \Gamma V(x(t), i, t) \\
&= \lim _{\Delta t \rightarrow 0} \frac{1}{\Delta t}\left[\mathscr { E } \left\{V\left(x(t+\Delta t), r_{t+\Delta t}, t+\Delta t\right) \mid x(t)=x,\right.\right. \\
&\left.\left.r_{t}=i\right\}-V(x(t), i, t)\right] \\
&=\frac{\partial}{\partial t} V(x(t), i, t)+\frac{\partial}{\partial x} V(x(t), i, t) \dot{x}(t) \\
& \quad+\sum_{j=1}^{N} \pi_{i j} V(x(t), j, t) .
\end{aligned}
$$


Lemma 6 (see [35]). Given constant matrices $\Omega_{1}, \Omega_{2}$, and $\Omega_{3}$, where $\Omega_{1}=\Omega_{1}^{T}$ and $\Omega_{2}=\Omega_{2}^{T}>0 . \Omega_{1}+\Omega_{3}^{T} \Omega_{2}^{-1} \Omega_{3}<0$ if and only if

$$
\left[\begin{array}{cc}
\Omega_{1} & \Omega_{3}^{T} \\
* & -\Omega_{2}
\end{array}\right]<0, \quad \text { or } \quad\left[\begin{array}{cc}
-\Omega_{2} & \Omega_{3}^{T} \\
* & \Omega_{1}
\end{array}\right]<0 .
$$

Lemma 7. For any constant matrix $Q=Q^{T}>0$, continuous functions $0 \leq h_{1}(t) \leq h_{2}(t)$, constant scalars $0 \leq \tau_{1}<\tau_{2}$, and constant $\varepsilon>0$ such that the following integrations are well defined,

(a)

$$
\begin{aligned}
& \frac{\exp \left\{2 \varepsilon h_{2}(t)\right\}-\exp \left\{2 \varepsilon h_{1}(t)\right\}}{2 \varepsilon} \\
& \quad \times \int_{t-h_{2}(t)}^{t-h_{1}(t)} \exp \{2 \varepsilon(s-t)\} x^{T}(s) Q x(s) d s \\
& \geq\left[\int_{t-h_{2}(t)}^{t-h_{1}(t)} x^{T}(s) d s\right] Q\left[\int_{t-h_{2}(t)}^{t-h_{1}(t)} x(s) d s\right] .
\end{aligned}
$$

(b)

$$
\begin{gathered}
\frac{\tau_{2}^{2}-\tau_{1}^{2}}{2} \int_{-\tau_{2}}^{-\tau_{1}} \int_{t+\theta}^{t} \exp \{2 \varepsilon(s-t)\} x^{T}(s) Q x(s) d s d \theta \\
\geq \exp \left\{-2 \varepsilon \tau_{2}\right\}\left[\int_{-\tau_{2}}^{-\tau_{1}} \int_{t+\theta}^{t} x^{T}(s) d s d \theta\right] \\
\times Q\left[\int_{-\tau_{2}}^{-\tau_{1}} \int_{t+\theta}^{t} x(s) d s d \theta\right] .
\end{gathered}
$$

Proof. (a) Is directly obtained from [36]. In addition, from $-\tau_{2} \leq \theta \leq-\tau_{1}$ and $t+\theta \leq s \leq t$, it is held that $-\tau_{2} \leq \theta \leq$ $s-t \leq 0$. Then

$$
\begin{aligned}
& \int_{-\tau_{2}}^{-\tau_{1}} \int_{t+\theta}^{t} \exp \{2 \varepsilon(s-t)\} x^{T}(s) Q x(s) d s d \theta \\
& \quad \geq \exp \left\{-2 \varepsilon \tau_{2}\right\} \int_{-\tau_{2}}^{-\tau_{1}} \int_{t+\theta}^{t} x^{T}(s) Q x(s) d s d \theta
\end{aligned}
$$

(b) Is thus true by [37].

Lemma 8 (see [38]). For functions $\lambda_{1}(t), \lambda_{2}(t) \in[0,1]$, $\lambda_{1}(t)+\lambda_{2}(t)=1$, and $\eta_{1}=0$ with $\lambda_{1}(t)=0$ and $\eta_{2}=0$ with $\lambda_{2}(t)=0$, matrices $P>0, Q>0$, then there exists matrix T such that

$$
\left[\begin{array}{cc}
P & T \\
T^{T} & Q
\end{array}\right]>0
$$

and the following inequality holds:

$$
\begin{aligned}
& \frac{1}{\lambda_{1}(t)} \eta_{1} P \eta_{1}^{T}+\frac{1}{\lambda_{2}(t)} \eta_{2} Q \eta_{2}^{T} \\
& \geq\left[\begin{array}{ll}
\eta_{1} & \eta_{2}
\end{array}\right]\left[\begin{array}{cc}
P & T \\
T^{T} & Q
\end{array}\right]\left[\begin{array}{l}
\eta_{1}^{T} \\
\eta_{2}^{T}
\end{array}\right] .
\end{aligned}
$$

Lemma 9 (see [39]). For given matrices $Q=Q^{T}, M$, and $N$ with appropriate dimensions,

$$
Q+M F(t) N+N^{T} F^{T}(t) M^{T}<0
$$

for all $F(t)$ satisfying $F^{T}(t) F(t) \leq I$, if and only if there exists a scalar $\delta>0$, such that

$$
Q+\delta^{-1} M M^{T}+\delta N N^{T}<0 .
$$

\section{Main Results}

This section will state the exponential stability analysis for neutral Markovian jump systems with mode-dependent interval time-varying delays, nonlinear perturbations, and partially known transition rates. With creative Lyapunov functional and novel matrix inequalities analysis, delayrange-dependent and rate-dependent exponential stability conditions are presented.

\subsection{Exponential Stability for the Nominal Systems}

Theorem 10. For given scalars $\pi_{i}^{m}, \pi_{i}^{M}, \alpha, \beta, \gamma, \varepsilon, \bar{\tau}_{i}, v_{i}, d_{1 i}$, $d_{2 i}, \mu_{i}$ and constant scalar $d_{m i}$ satisfying $d_{1 i}<d_{m i}<d_{2 i}$, the systems described by (13) with partially known transition rates are exponentially stable with decay rate $\varepsilon$ and $\kappa=\sqrt{\bar{\lambda}} / \underline{\lambda}$ if $\left\|C_{i}\right\|+\gamma<1$ and there exist symmetric positive matrices $P_{i}>$ $0, Q_{1 i}>0, Q_{2 i}>0, R_{1 i}>0, T_{1 i}>0,(i \in S), Q_{j}>0$, $(j=3,4), R_{k}>0, T_{l}>0,(k, l=2,3,4), U_{m}>0, V_{n}>0$, $W_{s}>0,(m, n, s=1,2,3,4)$ and matrices $M_{1}, M_{2}, N_{1}, N_{2}, N_{3}$, $N_{4}$ for any scalars $\varepsilon_{1}, \varepsilon_{2}, \varepsilon_{3}$, any symmetric matrices $X_{i}, Y_{1 i}$, $Y_{2 i}, Z_{1 i}, Z_{2 i},(i \in S)$ and any matrices $J_{k},(k=1,2, \ldots, 24)$ with appropriate dimensions, such that the following linear matrix inequalities hold.

When $i \in \mathcal{S}_{k}^{i}$

$$
\begin{gathered}
\sum_{j \in \mathcal{S}_{k}^{i}, j \neq i} \pi_{i j}\left(Q_{1 j}-Y_{1 i}\right)-\pi_{i i} Y_{1 i} \leq 0, \\
\sum_{j \in \delta_{k}^{i}, j \neq i} \pi_{i j}\left(Q_{2 j}-Y_{2 i}\right)-\pi_{i i} Y_{2 i} \leq 0, \\
\sum_{j \in \mathcal{S}_{k}^{i}, j \neq i} \pi_{i j}\left(R_{1 j}-Z_{1 i}\right)-\pi_{i i} Z_{1 i} \leq 0, \\
\sum_{j \in \mathcal{S}_{k}^{i}, j \neq i} \pi_{i j}\left(T_{1 j}-Z_{2 i}\right)-\pi_{i i} Z_{2 i} \leq 0, \\
P_{j}-X_{i} \leq 0, \quad j \in \mathcal{S}_{u k}^{i}, \\
Q_{1 j}-Y_{1 i} \leq 0, \quad j \in \mathcal{S}_{u k}^{i}, \\
Q_{2 j}-Y_{2 i} \leq 0, \quad j \in \mathcal{S}_{u k}^{i}, \\
R_{1 j}-Z_{1 i} \leq 0, \quad j \in \mathcal{S}_{u k}^{i}, \\
T_{1 j}-Z_{2 i} \leq 0, \quad j \in \mathcal{S}_{u k}^{i} .
\end{gathered}
$$


When $i \in \mathcal{S}_{u k}^{i}$

$$
\begin{gathered}
\sum_{j \in \mathcal{S}_{k}^{i}} \pi_{i j}\left(Q_{1 j}-Y_{1 i}\right) \leq 0 ; \quad \sum_{j \in \mathcal{S}_{k}^{i}} \pi_{i j}\left(Q_{2 j}-Y_{2 i}\right) \leq 0, \\
\sum_{j \in \mathcal{S}_{k}^{i}} \pi_{i j}\left(R_{1 j}-Z_{1 i}\right) \leq 0 ; \quad \sum_{j \in \mathcal{S}_{k}^{i}} \pi_{i j}\left(T_{1 j}-Z_{2 i}\right) \leq 0, \\
P_{j}-X_{i} \leq 0, \quad j \in \mathcal{S}_{u k}^{i}, \quad j \neq i, \\
-\sum_{j \in \mathcal{S}_{u k}^{i}, j \neq i} \pi_{i}^{M}\left(Q_{1 j}-Y_{1 i}\right)-\pi_{i}^{m} Y_{1 i} \leq 0, \\
-\sum_{j \in \mathcal{S}_{u k}^{i}, j \neq i} \pi_{i}^{M}\left(Q_{2 j}-Y_{2 i}\right)-\pi_{i}^{m} Y_{2 i} \leq 0, \\
-\sum_{j \in \delta_{u k}^{i}, j \neq i} \pi_{i}^{M}\left(R_{1 j}-Z_{1 i}\right)-\pi_{i}^{m} Z_{1 i} \leq 0, \\
-\sum_{j \in \mathcal{S}_{u k}^{i}, j \neq i} \pi_{i}^{M}\left(T_{1 j}-Z_{2 i}\right)-\pi_{i}^{m} Z_{2 i} \leq 0, \\
{\left[\begin{array}{ll}
U_{1} & M_{1} \\
M_{1}^{T} U_{1}
\end{array}\right]>0, \quad\left[\begin{array}{ll}
V_{1} & M_{2} \\
M_{2}^{T} & V_{1}
\end{array}\right]>0,} \\
(\mathrm{i}) \quad\left[\begin{array}{ll}
U_{3} & N_{1} \\
N_{1}^{T} & U_{3}
\end{array}\right]>0, \quad\left[\begin{array}{ll}
V_{3} \\
N_{2}^{T} & V_{3}
\end{array}\right]>0,
\end{gathered}
$$$$
\text { (ii) } \Omega_{i 0}^{\varepsilon}+\Omega_{i 1}^{\varepsilon}<0 \text {, }
$$$$
\text { (iii) }\left[\begin{array}{cc}
U_{4} & N_{3} \\
N_{3}^{T} & U_{4}
\end{array}\right]>0, \quad\left[\begin{array}{cc}
V_{4} & N_{4} \\
N_{4}^{T} & V_{4}
\end{array}\right]>0 \text {, }
$$

$$
\text { (iv) } \Omega_{i 0}^{\varepsilon}+\Omega_{i 2}^{\varepsilon}<0,
$$

where

$$
\begin{aligned}
& \Omega_{i 0}^{\varepsilon}=\sum_{m=1}^{24} e_{m} \Upsilon_{m} e_{m}^{T}+\mathscr{L}(\Xi) \\
& -\frac{2 \varepsilon d_{1 i}}{\exp \left\{2 \varepsilon d_{1 i}\right\}-1}\left(e_{1}-e_{5}\right) V_{2}\left(e_{1}^{T}-e_{5}^{T}\right) \\
& -\exp \left\{-2 \varepsilon \overline{\tau_{i}}\right\}\left(\overline{\tau_{i}} e_{1}-e_{21}\right) W_{1}\left(\overline{\tau_{i}} e_{1}^{T}-e_{21}^{T}\right) \\
& -\exp \left\{-2 \varepsilon d_{1 i}\right\}\left(d_{1 i} e_{1}-e_{11}\right) W_{2}\left(d_{1 i} e_{1}^{T}-e_{11}^{T}\right) \\
& -\exp \left\{-2 \varepsilon d_{m i}\right\}\left(\varrho_{1 i} e_{1}-e_{14}\right) W_{3}\left(\varrho_{1 i} e_{1}^{T}-e_{14}^{T}\right) \\
& -\exp \left\{-2 \varepsilon d_{2 i}\right\}\left(\varrho_{2 i} e_{1}-e_{15}\right) W_{4}\left(\varrho_{2 i} e_{1}^{T}-e_{15}^{T}\right) \\
& -\frac{2 \varepsilon \overline{\tau_{i}}}{\exp \left\{2 \varepsilon \overline{\tau_{i}}\right\}-1}\left[\begin{array}{ll}
e_{20} & e_{21}-e_{20}
\end{array}\right] \\
& \times\left[\begin{array}{cc}
U_{1} & M_{1} \\
M_{1}^{T} & U_{1}
\end{array}\right]\left[\begin{array}{c}
e_{20}^{T} \\
e_{21}^{T}-e_{20}^{T}
\end{array}\right]
\end{aligned}
$$

$$
\begin{aligned}
& -\frac{2 \varepsilon \overline{\tau_{i}}}{\exp \left\{2 \varepsilon \overline{\tau_{i}}\right\}-1}\left[\begin{array}{ll}
e_{1}-e_{18} & e_{18}-e_{16}
\end{array}\right] \\
& \times\left[\begin{array}{cc}
V_{1} & M_{2} \\
M_{2}^{T} & V_{1}
\end{array}\right]\left[\begin{array}{c}
e_{1}^{T}-e_{18}^{T} \\
e_{18}^{T}-e_{16}^{T}
\end{array}\right]
\end{aligned}
$$

where $\mathscr{L}$ is a linear operator on $\mathbb{R}^{n \times n}$ by

$$
\begin{aligned}
& \mathscr{L}(G)=G+G^{T}, \quad \forall G \in \mathbb{R}^{n \times n}, \\
& \Xi=-e_{1} J_{1} e_{2}^{T}+e_{1}\left(P_{i} B_{i}+J_{1} B_{i}\right) e_{3}^{T} \\
& +e_{1}\left(P_{i} C_{i}\right) e_{19}^{T}+\sum_{m=2}^{24} e_{1}\left(A_{i}^{T} J_{m}^{T}\right) e_{m}^{T} \\
& +e_{1} P_{i}\left(D_{i} e_{22}^{T}+E_{i} e_{23}^{T}+F_{i} e_{24}^{T}\right) \\
& +e_{2}\left(J_{2} B_{i}\right) e_{3}^{T}-\sum_{m=3}^{24} e_{2}\left(J_{m}^{T}\right) e_{m}^{T} \\
& +\sum_{m=4}^{24} e_{3}\left(B_{i}^{T} J_{m}^{T}\right) e_{m}^{T}+\sum_{m=1}^{18} e_{m}\left(J_{m} C_{i}\right) e_{19}^{T} \\
& +\sum_{m=20}^{24} e_{19}\left(C_{i}^{T} J_{m}^{T}\right) e_{m}^{T} \\
& +\sum_{m=1}^{21} e_{m} J_{m}\left(D_{i} e_{22}^{T}+E_{i} e_{23}^{T}+F_{i} e_{24}^{T}\right) \\
& +e_{22} J_{22}\left(E_{i} e_{23}^{T}+F_{i} e_{24}^{T}\right)+\sum_{m=23}^{24} e_{22}\left(D_{i}^{T} J_{m}^{T}\right) e_{m}^{T} \\
& +e_{23}\left(J_{23} F_{i}\right) e_{24}^{T}+e_{23}\left(E_{i}^{T} J_{24}^{T}\right) e_{24}^{T}, \\
& \Upsilon_{1}=A_{i}^{T} P_{i}+P_{i} A_{i}+\sum_{j \in \delta_{k}^{i}} \pi_{i j}\left(P_{j}-X_{i}\right) \\
& +2 \varepsilon P_{i}+Q_{1 i}+Q_{3}+R_{2}+\bar{\tau}_{i}^{2} U_{1}+d_{1 i}^{2} U_{2} \\
& +\varrho_{1 i}^{2} U_{3}+\varrho_{2 i}^{2} U_{4}+\varepsilon_{1} \alpha^{2} I+J_{1} A_{i}+A_{i}^{T} J_{1}^{T}, \\
& \Upsilon_{2}=Q_{2 i}+Q_{4}+T_{2}+\bar{\tau}_{i}^{2} V_{1}+d_{1 i}^{2} V_{2} \\
& +\varrho_{1 i}^{2} V_{3}+\varrho_{2 i}^{2} V_{4}+\frac{\bar{\tau}_{i}^{4}}{4} W_{1}+\frac{d_{1 i}^{4}}{4} W_{2} \\
& +\varrho_{3 i}^{2} W_{3}+\varrho_{4 i}^{2} W_{4}-J_{2}-J_{2}^{T}, \\
& \Upsilon_{3}=\omega\left(\varepsilon, \mu_{i}\right) R_{1 i}+\varepsilon_{2} \beta^{2} I+J_{3} B_{i}+B_{i}^{T} J_{3}^{T} \text {, } \\
& \Upsilon_{4}=\emptyset\left(\varepsilon, \mu_{i}\right) T_{1 i} \text {, } \\
& \Upsilon_{5}=\exp \left\{-2 \varepsilon d_{1 i}\right\}\left(R_{1 i}+R_{3}-R_{2}\right), \\
& \Upsilon_{6}=\exp \left\{-2 \varepsilon d_{m i}\right\}\left(R_{4}-R_{3}\right) \text {, } \\
& \Upsilon_{7}=-\exp \left\{-2 \varepsilon d_{2 i}\right\} R_{4} \text {, }
\end{aligned}
$$




$$
\begin{aligned}
& \Upsilon_{8}=\exp \left\{-2 \varepsilon d_{1 i}\right\}\left(T_{1 i}+T_{3}-T_{2}\right) \\
& \Upsilon_{9}=\exp \left\{-2 \varepsilon d_{m i}\right\}\left(T_{4}-T_{3}\right), \\
& \Upsilon_{10}=-\exp \left\{-2 \varepsilon d_{2 i}\right\} T_{4} \text {, } \\
& \Upsilon_{11}=-\frac{2 \varepsilon d_{1 i}}{\exp \left\{2 \varepsilon d_{1 i}\right\}-1} U_{2}, \\
& \Upsilon_{16}=-\exp \left\{-2 \varepsilon \overline{\tau_{i}}\right\} Q_{3} \text {, } \\
& \Upsilon_{17}=-\exp \left\{-2 \varepsilon \overline{\tau_{i}}\right\} Q_{4} \text {, } \\
& \Upsilon_{18}=-\left(1-v_{i}\right) \exp \left\{-2 \varepsilon \overline{\tau_{i}}\right\} Q_{1 i} \text {, } \\
& \Upsilon_{19}=-\left(1-v_{i}\right) \exp \left\{-2 \varepsilon \overline{\tau_{i}}\right\} Q_{2 i} \\
& +\varepsilon_{3} \gamma^{2} I+J_{19} C_{i}+C_{i}^{T} J_{19}^{T} \text {, } \\
& \Upsilon_{22}=-\varepsilon_{1} I+J_{22} D_{i}+D_{i}^{T} J_{22}^{T}, \\
& \Upsilon_{23}=-\varepsilon_{2} I+J_{23} E_{i}+E_{i}^{T} J_{23}^{T} \text {, } \\
& \Upsilon_{24}=-\varepsilon_{3} I+J_{24} F_{i}+F_{i}^{T} J_{24}^{T}, \\
& \Upsilon_{m}=0, \quad(m=12,13,14,15,20,21) \text {, } \\
& \Omega_{i 1}^{\varepsilon}=-\frac{2 \varepsilon \varrho_{2 i}}{\exp \left\{2 \varepsilon d_{2 i}\right\}-\exp \left\{2 \varepsilon d_{m i}\right\}} e_{15} U_{4} e_{15}^{T} \\
& -\frac{2 \varepsilon \varrho_{2 i}}{\exp \left\{2 \varepsilon d_{2 i}\right\}-\exp \left\{2 \varepsilon d_{m i}\right\}} \\
& \times\left(e_{6}-e_{7}\right) V_{4}\left(e_{6}^{T}-e_{7}^{T}\right)
\end{aligned}
$$

$$
\begin{aligned}
& -\frac{2 \varepsilon \varrho_{1 i}}{\exp \left\{2 \varepsilon d_{m i}\right\}-\exp \left\{2 \varepsilon d_{1 i}\right\}}\left[\begin{array}{ll}
e_{12} & e_{14}-e_{12}
\end{array}\right] \\
& \times\left[\begin{array}{cc}
U_{3} & N_{1} \\
N_{1}^{T} & U_{3}
\end{array}\right]\left[\begin{array}{c}
e_{12}^{T} \\
e_{14}^{T}-e_{12}^{T}
\end{array}\right] \\
& -\frac{2 \varepsilon Q_{1 i}}{\exp \left\{2 \varepsilon d_{m i}\right\}-\exp \left\{2 \varepsilon d_{1 i}\right\}}\left[e_{5}-e_{3} e_{3}-e_{6}\right] \\
& \times\left[\begin{array}{cc}
V_{3} & N_{2} \\
N_{2}^{T} & V_{3}
\end{array}\right]\left[\begin{array}{c}
e_{5}^{T}-e_{3}^{T} \\
e_{3}^{T}-e_{6}^{T}
\end{array}\right], \\
& \Omega_{i 2}^{\varepsilon}=-\frac{2 \varepsilon \varrho_{1 i}}{\exp \left\{2 \varepsilon d_{m i}\right\}-\exp \left\{2 \varepsilon d_{1 i}\right\}} e_{14} U_{3} e_{14}^{T} \\
& -\frac{2 \varepsilon \varrho_{1 i}}{\exp \left\{2 \varepsilon d_{m i}\right\}-\exp \left\{2 \varepsilon d_{1 i}\right\}} \\
& \times\left(e_{5}-e_{6}\right) V_{3}\left(e_{5}^{T}-e_{6}^{T}\right) \\
& -\frac{2 \varepsilon \varrho_{2 i}}{\exp \left\{2 \varepsilon d_{2 i}\right\}-\exp \left\{2 \varepsilon d_{m i}\right\}}\left[\begin{array}{ll}
e_{13} & e_{15}-e_{13}
\end{array}\right] \\
& \times\left[\begin{array}{cc}
U_{4} & N_{3} \\
N_{3}^{T} & U_{4}
\end{array}\right]\left[\begin{array}{c}
e_{13}^{T} \\
e_{15}^{T}-e_{13}^{T}
\end{array}\right] \\
& -\frac{2 \varepsilon \varrho_{2 i}}{\exp \left\{2 \varepsilon d_{2 i}\right\}-\exp \left\{2 \varepsilon d_{m i}\right\}}\left[\begin{array}{ll}
e_{3}-e_{7} & e_{6}-e_{3}
\end{array}\right] \\
& \times\left[\begin{array}{cc}
V_{4} & N_{4} \\
N_{4}^{T} & V_{4}
\end{array}\right]\left[\begin{array}{c}
e_{3}^{T}-e_{7}^{T} \\
e_{6}^{T}-e_{3}^{T}
\end{array}\right],
\end{aligned}
$$

where $e_{i}\{i=1,2, \ldots, 24\}$ are block entry matrices; that is,

$$
\begin{aligned}
& e_{3}^{T}=\left[\begin{array}{llllllllllllllllllllllll}
0 & 0 & I & 0 & 0 & 0 & 0 & 0 & 0 & 0 & 0 & 0 & 0 & 0 & 0 & 0 & 0 & 0 & 0 & 0 & 0 & 0 & 0 & 0
\end{array}\right] \\
& \omega\left(\varepsilon, \mu_{i}\right)=\left(\mu_{i}-1\right) \exp \left\{-2 \varepsilon d_{1 i}\right\} \quad \text { with } \mu_{i} \geq 1, \quad\left(\mu_{i}-1\right) \exp \left\{-2 \varepsilon d_{2 i}\right\} \quad \text { with } \mu_{i}<1, \\
& \bar{\lambda}=\max _{i \in S}\left\{\lambda_{\max }\left(P_{i}\right)\right\}+\frac{1-\exp \{-2 \varepsilon \varsigma\}}{2 \varepsilon}\left\{\sum_{j=3}^{4} \lambda_{\max }\left(Q_{j}\right)+\sum_{k=2}^{4} \lambda_{\max }\left(R_{k}\right)+\sum_{l=2}^{4} \lambda_{\max }\left(T_{l}\right)\right\} \\
& +\frac{1-\exp \{-2 \varepsilon \varsigma\}}{2 \varepsilon}\left\{\max _{i \in S}\left\{\lambda_{\max }\left(Q_{1 i}\right)\right\}+\max _{i \in S}\left\{\lambda_{\max }\left(Q_{2 i}\right)\right\}+\max _{i \in S}\left\{\lambda_{\max }\left(R_{1 i}\right)\right\}+\max _{i \in S}\left\{\lambda_{\max }\left(T_{1 i}\right)\right\}\right\} \\
& +\frac{\exp \{-2 \varepsilon \varsigma\}+2 \varepsilon \varsigma-1}{4 \varepsilon^{2}}\left\{\sum_{m=1}^{4} \lambda_{\max }\left(U_{m}\right)+\sum_{n=1}^{4} \lambda_{\max }\left(V_{n}\right)\right\} \\
& +\frac{1-\exp \{-2 \varepsilon \varsigma\}+2 \varepsilon^{2} \varsigma^{2}-2 \varepsilon \varsigma}{8 \varepsilon^{3}}\left\{\sum_{s=1}^{4} \lambda_{\max }\left(W_{s}\right)\right\} \\
& \underline{\lambda}=\min _{i \in S}\left\{\lambda_{\min }\left(P_{i}\right)\right\}, \quad \varrho_{1 i}=d_{m i}-d_{1 i}, \quad \varrho_{2 i}=d_{2 i}-d_{m i}, \quad \varrho_{3 i}=\frac{1}{2}\left(d_{m i}^{2}-d_{1 i}^{2}\right), \quad \varrho_{4 i}=\frac{1}{2}\left(d_{2 i}^{2}-d_{m i}^{2}\right) .
\end{aligned}
$$


Proof. Construct the following stochastic Lyapunov functional:

$$
V(x(t), i, t)=\sum_{k=1}^{7} V_{k}\left(x_{t}, i\right),
$$

where

$$
\begin{aligned}
& V_{1}\left(x_{t}, i\right)=x^{T}(t) P_{i} x(t), \\
& V_{2}\left(x_{t}, i\right)=\int_{t-\tau_{i}(t)}^{t} \exp \{2 \varepsilon(s-t)\} x^{T}(s) Q_{1 i} x(s) d s \\
& +\int_{t-\tau_{i}(t)}^{t} \exp \{2 \varepsilon(s-t)\} \dot{x}^{T}(s) Q_{2 i} \dot{x}(s) d s \\
& +\int_{t-\bar{\tau}_{i}}^{t} \exp \{2 \varepsilon(s-t)\} x^{T}(s) Q_{3} x(s) d s \\
& +\int_{t-\overline{\tau_{i}}}^{t} \exp \{2 \varepsilon(s-t)\} \dot{x}^{T}(s) Q_{4} \dot{x}(s) d s, \\
& V_{3}\left(x_{t}, i\right)=\int_{t-d_{i}(t)}^{t-d_{1 i}} \exp \{2 \varepsilon(s-t)\} x^{T}(s) R_{1 i} x(s) d s \\
& +\int_{t-d_{1 i}}^{t} \exp \{2 \varepsilon(s-t)\} x^{T}(s) R_{2} x(s) d s \\
& +\int_{t-d_{m i}}^{t-d_{1 i}} \exp \{2 \varepsilon(s-t)\} x^{T}(s) R_{3} x(s) d s \\
& +\int_{t-d_{2 i}}^{t-d_{m i}} \exp \{2 \varepsilon(s-t)\} x^{T}(s) R_{4} x(s) d s, \\
& V_{4}\left(x_{t}, i\right)=\int_{t-d_{i}(t)}^{t-d_{1 i}} \exp \{2 \varepsilon(s-t)\} \dot{x}^{T}(s) T_{1 i} \dot{x}(s) d s \\
& +\int_{t-d_{1 i}}^{t} \exp \{2 \varepsilon(s-t)\} \dot{x}^{T}(s) T_{2} \dot{x}(s) d s \\
& +\int_{t-d_{m i}}^{t-d_{1 i}} \exp \{2 \varepsilon(s-t)\} \dot{x}^{T}(s) T_{3} \dot{x}(s) d s \\
& +\int_{t-d_{2 i}}^{t-d_{m i}} \exp \{2 \varepsilon(s-t)\} \dot{x}^{T}(s) T_{4} \dot{x}(s) d s, \\
& V_{5}\left(x_{t}, i\right)=\int_{-\overline{\tau_{i}}}^{0} \int_{t+\theta}^{t} \overline{\tau_{i}} \exp \{2 \varepsilon(s-t)\} x^{T}(s) U_{1} x(s) d s d \theta \\
& +\int_{-d_{1 i}}^{0} \int_{t+\theta}^{t} d_{1 i} \exp \{2 \varepsilon(s-t)\} x^{T} \\
& \times(s) U_{2} x(s) d s d \theta \\
& +\int_{-d_{m i}}^{-d_{1 i}} \int_{t+\theta}^{t} \varrho_{1 i} \exp \{2 \varepsilon(s-t)\} x^{T} \\
& \times(s) U_{3} x(s) d s d \theta \\
& +\int_{-d_{2 i}}^{-d_{m i}} \int_{t+\theta}^{t} \varrho_{2 i} \exp \{2 \varepsilon(s-t)\} x^{T} \\
& \times(s) U_{4} x(s) d s d \theta,
\end{aligned}
$$

$$
\begin{aligned}
V_{6}\left(x_{t}, i\right)= & \int_{-\bar{\tau}_{i}}^{0} \int_{t+\theta}^{t} \overline{\tau_{i}} \exp \{2 \varepsilon(s-t)\} \dot{x}^{T}(s) V_{1} \dot{x}(s) d s d \theta \\
& +\int_{-d_{1 i}}^{0} \int_{t+\theta}^{t} d_{1 i} \exp \{2 \varepsilon(s-t)\} \dot{x}^{T} \\
& \times(s) V_{2} \dot{x}(s) d s d \theta \\
& +\int_{-d_{m i}}^{-d_{1 i}} \int_{t+\theta}^{t} \varrho_{1 i} \exp \{2 \varepsilon(s-t)\} \dot{x}^{T} \\
& \times(s) V_{3} \dot{x}(s) d s d \theta \\
& +\int_{-d_{2 i}}^{-d_{m i}} \int_{t+\theta}^{t} \varrho_{2 i} \exp \{2 \varepsilon(s-t)\} \dot{x}^{T} \\
& \times(s) V_{4} \dot{x}(s) d s d \theta,
\end{aligned}
$$$$
V_{7}\left(x_{t}, i\right)=\int_{-\bar{\tau}_{i}}^{0} \int_{\theta}^{0} \int_{t+\lambda}^{t} \frac{\bar{\tau}_{i}^{2}}{2} \exp \{2 \varepsilon(s-t)\} \dot{x}^{T}
$$$$
\times(s) W_{1} \dot{x}(s) d s d \lambda d \theta
$$$$
+\int_{-d_{1 i}}^{0} \int_{\theta}^{0} \int_{t+\lambda}^{t} \frac{d_{1 i}^{2}}{2} \exp \{2 \varepsilon(s-t)\}
$$$$
\times \dot{x}^{T}(s) W_{2} \dot{x}(s) d s d \lambda d \theta
$$$$
+\int_{-d_{m i}}^{-d_{1 i}} \int_{\theta}^{0} \int_{t+\lambda}^{t} \varrho_{3 i} \exp \{2 \varepsilon(s-t)\}
$$$$
\times \dot{x}^{T}(s) W_{3} \dot{x}(s) d s d \lambda d \theta
$$$$
+\int_{-d_{2 i}}^{-d_{m i}} \int_{\theta}^{0} \int_{t+\lambda}^{t} \varrho_{4 i} \exp \{2 \varepsilon(s-t)\}
$$$$
\times \dot{x}^{T}(s) W_{4} \dot{x}(s) d s d \lambda d \theta .
$$

Remark 11. It should be pointed out that the proposed stochastic augmented Lyapunov functional (38) contains some triple-integral terms, which has not been used in of the existing literature in the same context before. Compared with the existing ones, [37] has shown that such triple-integral terms are very effective in the reduction of conservativeness.

Taking $\Gamma$ as its infinitesimal generator along the trajectory of system (13), we obtain the following from Definition 5 and (38)-(45):

$$
\Gamma V(x(t), i, t)=\sum_{k=1}^{7} \Gamma V_{k}\left(x_{t}, i\right),
$$

$\Gamma V_{1}\left(x_{t}, i\right)$ can be easily obtained by the following equation:

$$
\begin{aligned}
& \Gamma V_{1}\left(x_{t}, i\right)=2\left[x^{T}(t) A_{i}^{T}+x^{T}\left(t-d_{i}(t)\right) B_{i}^{T}\right. \\
&+\dot{x}^{T}\left(t-\tau_{i}(t)\right) C_{i}^{T}+f_{1}^{T} D_{i}^{T} \\
&\left.\quad+f_{2}^{T} E_{i}^{T}+f_{3}^{T} F_{i}^{T}\right] \\
& \times P_{i} x(t)+x^{T}(t)\left[\sum_{j \in \delta_{k}^{i}} \pi_{i j} P_{j}+\sum_{j \in \delta_{u k}^{i}} \pi_{i j} P_{j}\right] x(t) \\
&+2 \varepsilon x^{T}(t) P_{i} x(t)-2 \varepsilon V_{1}\left(x_{t}, i\right) .
\end{aligned}
$$


With regard to $\Gamma V_{2}\left(x_{t}, i\right)$, the detailed procedures are given as follows.

Define $V_{2}\left(x_{t}, i\right)=V_{21}\left(x_{t}, i\right)+V_{22}\left(x_{t}, i\right)$

$$
+V_{23}\left(x_{t}, i\right)+V_{24}\left(x_{t}, i\right)
$$

where

$$
\begin{aligned}
& V_{21}\left(x_{t}, i\right)=\int_{t-\tau_{i}(t)}^{t} \exp \{2 \varepsilon(s-t)\} x^{T}(s) Q_{1 i} x(s) d s, \\
& V_{22}\left(x_{t}, i\right)=\int_{t-\tau_{i}(t)}^{t} \exp \{2 \varepsilon(s-t)\} \dot{x}^{T}(s) Q_{2 i} \dot{x}(s) d s, \\
& V_{23}\left(x_{t}, i\right)=\int_{t-\overline{\tau_{i}}}^{t} \exp \{2 \varepsilon(s-t)\} x^{T}(s) Q_{3} x(s) d s, \\
& V_{24}\left(x_{t}, i\right)=\int_{t-\overline{\tau_{i}}}^{t} \exp \{2 \varepsilon(s-t)\} \dot{x}^{T}(s) Q_{4} \dot{x}(s) d s .
\end{aligned}
$$

By the infinitesimal generator $\Gamma$, we obtain

$$
\begin{aligned}
\Gamma V_{21}\left(x_{t}, i\right)= & \exp \{-2 \varepsilon t\} \\
& \times \Gamma\left(\int_{t-\tau_{i}(t)}^{t} \exp \{2 \varepsilon s\} x^{T}(s) Q_{1 i} x(s) d s\right) \\
& -2 \varepsilon V_{21}\left(x_{t}, i\right),
\end{aligned}
$$

where

$$
\begin{gathered}
\Gamma\left(\int_{t-\tau_{i}(t)}^{t} \exp \{2 \varepsilon s\} x^{T}(s) Q_{1 i} x(s) d s\right) \\
=\lim _{\Delta \rightarrow 0^{+}} \frac{1}{\Delta}\left\{\mathscr { E } \left[\int_{t+\Delta-\tau\left(t+\Delta, r_{t+\Delta}\right)}^{t+\Delta} \exp \{2 \varepsilon s\} x^{T}(s) Q_{1}\left(r_{t+\Delta}\right)\right.\right. \\
\left.\times x(s) d s \mid x(t), r_{t}=i\right] \\
=\lim _{\Delta \rightarrow 0^{+}} \frac{1}{\Delta}\left\{\sum_{j=1}^{N}\left(\int_{t-\tau_{i}(t)}^{t} \exp \{2 \varepsilon s\} x^{T}(s) Q_{1 i} x(s) d s\right\}\right. \\
\times\left[\int_{t+\Delta-\tau_{j}(t+\Delta)}^{t+\Delta} \exp \{2 \varepsilon s\} x^{T}(s) Q_{1 j} x(s) d s\right] \\
\left.-\int_{t-\tau_{i}(t)}^{t} \exp \{2 \varepsilon s\} x^{T}(s) Q_{1 i} x(s) d s\right\} \\
\lim _{\Delta \rightarrow 0^{+}} \frac{1}{\Delta}\left[\left(\sum_{j=1}^{N} \pi_{i j} \Delta\right) \int_{t+\Delta-\tau_{j}(t+\Delta)}^{t+\Delta} \times Q_{1 j} x(s) d s\right. \\
\exp \{2 \varepsilon s\} x^{T}(s)
\end{gathered}
$$

$$
\begin{aligned}
& +\int_{t+\Delta-\tau_{i}(t+\Delta)}^{t+\Delta} \exp \{2 \varepsilon s\} x^{T}(s) Q_{1 i} x(s) d s \\
& \left.-\int_{t-\tau_{i}(t)}^{t} \exp \{2 \varepsilon s\} x^{T}(s) Q_{1 i} x(s) d s\right]
\end{aligned}
$$

$$
\begin{aligned}
= & \sum_{j=1}^{N} \pi_{i j} \int_{t-\tau_{j}(t)}^{t} \exp \{2 \varepsilon s\} x^{T}(s) Q_{1 j} x(s) d s \\
& +\exp \{2 \varepsilon(t)\} x^{T}(t) Q_{1 i} x(t) \\
& -\left(1-\dot{\tau}_{i}(t)\right) \exp \left\{2 \varepsilon\left(t-\tau_{i}(t)\right)\right\} x^{T} \\
& \times\left(t-\tau_{i}(t)\right) Q_{1 i} x\left(t-\tau_{i}(t)\right) .
\end{aligned}
$$

Following the same procedure, $\Gamma V_{22}\left(x_{t}, i\right)$ is also obtained:

$$
\begin{aligned}
\Gamma V_{22}\left(x_{t}, i\right)= & \dot{x}^{T}(t) Q_{2 i} \dot{x}(t)-\left(1-\dot{\tau}_{i}(t)\right) \exp \left\{2 \varepsilon\left(t-\tau_{i}(t)\right)\right\} \\
& \times \dot{x}^{T}\left(t-\tau_{i}(t)\right) Q_{2 i} \dot{x}\left(t-\tau_{i}(t)\right) \\
& -\sum_{j=1}^{N} \pi_{i j} \int_{t-\tau_{j}(t)}^{t} \exp \{2 \varepsilon s\} \dot{x}^{T}(s) Q_{2 j} \dot{x}(s) d s \\
& -2 \varepsilon V_{22}\left(x_{t}, i\right) .
\end{aligned}
$$

Moreover, $\Gamma V_{23}\left(x_{t}, i\right), \Gamma V_{24}\left(x_{t}, i\right)$ are easily calculated as shown in the following:

$$
\begin{aligned}
\Gamma V_{23}\left(x_{t}, i\right)= & x^{T}(t) Q_{3} x(t)-\exp \left\{-2 \varepsilon \overline{\tau_{i}}\right\} \\
& \times x^{T}\left(t-\overline{\tau_{i}}\right) Q_{3} x\left(t-\overline{\tau_{i}}\right)-2 \varepsilon V_{23}\left(x_{t}, i\right), \\
\Gamma V_{24}\left(x_{t}, i\right)= & \dot{x}^{T}(t) Q_{4} \dot{x}(t)-\exp \left\{-2 \varepsilon \overline{\tau_{i}}\right\} \\
& \times \dot{x}^{T}\left(t-\overline{\tau_{i}}\right) Q_{4} \dot{x}\left(t-\overline{\tau_{i}}\right)-2 \varepsilon V_{24}\left(x_{t}, i\right) .
\end{aligned}
$$

According to (50), (51), (52), and (53), we can easily obtain the following (54):

$$
\begin{aligned}
\Gamma V_{2}( & \left.x_{t}, i\right) \\
= & x^{T}(t)\left[Q_{1 i}+Q_{3}\right] x(t) \\
& +\dot{x}^{T}(t)\left[Q_{2 i}+Q_{4}\right] \dot{x}(t)-2 \varepsilon V_{2}\left(x_{t}, i\right) \\
& -\left(1-\dot{\tau}_{i}(t)\right) \exp \left\{-2 \varepsilon \tau_{i}(t)\right\} x^{T}\left(t-\tau_{i}(t)\right) Q_{1 i} x \\
& \times\left(t-\tau_{i}(t)\right)-\left(1-\dot{\tau}_{i}(t)\right) \exp \left\{-2 \varepsilon \tau_{i}(t)\right\} \dot{x}^{T} \\
& \times\left(t-\tau_{i}(t)\right) Q_{2 i} \dot{x}\left(t-\tau_{i}(t)\right) \\
& -\exp \left\{-2 \varepsilon \overline{\tau_{i}}\right\} x^{T}\left(t-\overline{\tau_{i}}\right) Q_{3} x\left(t-\overline{\tau_{i}}\right) \\
& -\exp \left\{-2 \varepsilon \overline{\tau_{i}}\right\} \dot{x}^{T}\left(t-\overline{\tau_{i}}\right) Q_{4} \dot{x}\left(t-\overline{\tau_{i}}\right) \\
& +\sum_{j \in S} \pi_{i j} \int_{t-\tau_{j}(t)}^{t} \exp \{2 \varepsilon(s-t)\} \\
& \times\left[x^{T}(s) Q_{1 j} x(s)+\dot{x}^{T}(s) Q_{2 j} \dot{x}(s)\right] d s
\end{aligned}
$$




$$
\begin{aligned}
\leq & x^{T}(t)\left[Q_{1 i}+Q_{3}\right] x(t) \\
+ & \dot{x}^{T}(t)\left[Q_{2 i}+Q_{4}\right] \dot{x}(t)-2 \varepsilon V_{2}\left(x_{t}, i\right) \\
- & \left(1-\dot{\tau}_{i}(t)\right) \exp \left\{-2 \varepsilon \tau_{i}(t)\right\} x^{T}\left(t-\tau_{i}(t)\right) Q_{1 i} x \\
& \times\left(t-\tau_{i}(t)\right)-\left(1-\dot{\tau}_{i}(t)\right) \exp \left\{-2 \varepsilon \tau_{i}(t)\right\} \dot{x}^{T} \\
& \times\left(t-\tau_{i}(t)\right) Q_{2 i} \dot{x}\left(t-\tau_{i}(t)\right) \\
- & \exp \left\{-2 \varepsilon \overline{\tau_{i}}\right\} x^{T}\left(t-\overline{\tau_{i}}\right) Q_{3} x\left(t-\overline{\tau_{i}}\right) \\
- & \exp \left\{-2 \varepsilon \overline{\tau_{i}}\right\} \dot{x}^{T}\left(t-\overline{\tau_{i}}\right) Q_{4} \dot{x}\left(t-\overline{\tau_{i}}\right) \\
+ & \sum_{j \neq i} \pi_{i j} \int_{t-\bar{\tau}_{j}}^{t} \exp \{2 \varepsilon(s-t)\} \\
& \quad \times\left[x^{T}(s) Q_{1 j} x(s)+\dot{x}^{T}(s) Q_{2 j} \dot{x}(s)\right] d s .
\end{aligned}
$$

Then, in the same method, $\Gamma V_{3}\left(x_{t}, i\right), \Gamma V_{4}\left(x_{t}, i\right)$ can be calculated and the results are given by the following, respectively:

$$
\begin{aligned}
\Gamma V_{3}( & \left.x_{t}, i\right) \\
= & x^{T}(t) R_{2} x(t)+\exp \left\{-2 \varepsilon d_{1 i}\right\} x^{T} \\
& \times\left(t-d_{1 i}\right)\left[R_{1 i}+R_{3}-R_{2}\right] x\left(t-d_{1 i}\right) \\
& +\exp \left\{-2 \varepsilon d_{m i}\right\} x^{T}\left(t-d_{m i}\right)\left[R_{4}-R_{3}\right] x\left(t-d_{m i}\right) \\
& -\exp \left\{-2 \varepsilon d_{2 i}\right\} x^{T}\left(t-d_{2 i}\right) R_{4} x\left(t-d_{2 i}\right) \\
& -2 \varepsilon V_{3}\left(x_{t}, i\right)-\left(1-\dot{d}_{i}(t)\right) \\
& \times \exp \left\{-2 \varepsilon d_{i}(t)\right\} x^{T}\left(t-d_{i}(t)\right) R_{1 i} x\left(t-d_{i}(t)\right) \\
& +\sum_{j \in S} \pi_{i j} \int_{t-d_{j}(t)}^{t-d_{1 i}} \exp \{2 \varepsilon(s-t)\} x^{T}(s) R_{1 j} x(s) d s \\
\leq & x^{T}(t) R_{2} x(t)+\exp \left\{-2 \varepsilon d_{1 i}\right\} x^{T}\left(t-d_{1 i}\right) \\
& \times\left[R_{1 i}+R_{3}-R_{2}\right] x\left(t-d_{1 i}\right) \\
& +\exp \left\{-2 \varepsilon d_{m i}\right\} x^{T}\left(t-d_{m i}\right)\left[R_{4}-R_{3}\right] x \\
& \times\left(t-d_{m i}\right)-\exp \left\{-2 \varepsilon d_{2 i}\right\} x^{T} \\
& \times\left(t-d_{2 i}\right) R_{4} x\left(t-d_{2 i}\right)-2 \varepsilon V_{3}\left(x_{t}, i\right) \\
& -\left(1-\dot{d}_{i}(t)\right) \exp \left\{-2 \varepsilon d_{i}(t)\right\} x^{T} \\
& \times\left(t-d_{i}(t)\right) R_{1 i} x\left(t-d_{i}(t)\right) \\
& +\sum_{j \neq i} \pi_{i j} \int_{t-d_{2 j}}^{t-d_{1 i}} \exp \{2 \varepsilon(s-t)\} x^{T}(s) R_{1 j} x(s) d s,
\end{aligned}
$$

$$
\begin{aligned}
\Gamma V_{4}\left(x_{t}, i\right) \\
=\dot{x}^{T}(t) T_{2} \dot{x}(t)+\exp \left\{-2 \varepsilon d_{1 i}\right\} \dot{x}^{T} \\
\quad \times\left(t-d_{1 i}\right)\left[T_{1 i}+T_{3}-T_{2}\right] \dot{x}\left(t-d_{1 i}\right) \\
\quad+\exp \left\{-2 \varepsilon d_{m i}\right\} \dot{x}^{T}\left(t-d_{m i}\right) \\
\quad \times\left[T_{4}-T_{3}\right] \dot{x}\left(t-d_{m i}\right) \\
\quad-\exp \left\{-2 \varepsilon d_{2 i}\right\} \dot{x}^{T}\left(t-d_{2 i}\right) T_{4} \dot{x}\left(t-d_{2 i}\right) \\
\quad-2 \varepsilon V_{4}\left(x_{t}, i\right)-\left(1-\dot{d}_{i}(t)\right) \exp \left\{-2 \varepsilon d_{i}(t)\right\} \dot{x}^{T} \\
\quad \times\left(t-d_{i}(t)\right) T_{1 i} \dot{x}\left(t-d_{i}(t)\right) \\
+\sum_{j \in S} \pi_{i j} \int_{t-d_{j}(t)}^{t-d_{1 i}} \exp \{2 \varepsilon(s-t)\} \dot{x}^{T}(s) T_{1 j} \dot{x}(s) d s \\
\leq \dot{x}^{T}(t) T_{2} \dot{x}(t)+\exp \left\{-2 \varepsilon d_{1 i}\right\} \dot{x}^{T}\left(t-d_{1 i}\right) \\
\quad \times\left[T_{1 i}+T_{3}-T_{2}\right] \dot{x}\left(t-d_{1 i}\right) \\
\quad+\exp \left\{-2 \varepsilon d_{m i}\right\} \dot{x}^{T}\left(t-d_{m i}\right)\left[T_{4}-T_{3}\right] \dot{x}\left(t-d_{m i}\right) \\
\quad-\exp \left\{-2 \varepsilon d_{2 i}\right\} \dot{x}^{T}\left(t-d_{2 i}\right) T_{4} \dot{x}\left(t-d_{2 i}\right) \\
\quad-2 \varepsilon V_{4}\left(x_{t}, i\right)-\left(1-\dot{d}_{i}(t)\right) \\
\quad \times \exp \left\{-2 \varepsilon d_{i}(t)\right\} \dot{x}^{T}\left(t-d_{i}(t)\right) T_{1 i} \dot{x}\left(t-d_{i}(t)\right) \\
+\sum_{j \neq i} \pi_{i j} \int_{t-d_{2 j}}^{t-d_{1 i}} \exp \{2 \varepsilon(s-t)\} \dot{x}^{T}(s) T_{1 j} \dot{x}(s) d s .
\end{aligned}
$$

Moreover, $\Gamma V_{5}\left(x_{t}, i\right), \Gamma V_{6}\left(x_{t}, i\right)$, and $\Gamma V_{7}\left(x_{t}, i\right)$ can be directly obtained as follows:

$$
\begin{aligned}
\Gamma V_{5}\left(x_{t}, i\right) \\
=x^{T}(t)\left[\bar{\tau}_{i}^{2} U_{1}+d_{1 i}^{2} U_{2}+\varrho_{1 i}^{2} U_{3}+\varrho_{2 i}^{2} U_{4}\right] x(t) \\
\quad-2 \varepsilon V_{5}\left(x_{t}, i\right) \\
\quad-\int_{t-\overline{\tau_{i}}}^{t} \overline{\tau_{i}} \exp \{2 \varepsilon(s-t)\} x^{T}(s) U_{1} x(s) d s \\
-\int_{t-d_{1 i}}^{t} d_{1 i} \exp \{2 \varepsilon(s-t)\} x^{T}(s) U_{2} x(s) d s \\
\quad-\int_{t-d_{m i}}^{t-d_{1 i}} \varrho_{1 i} \exp \{2 \varepsilon(s-t)\} x^{T}(s) U_{3} x(s) d s \\
\quad-\int_{t-d_{2 i}}^{t-d_{m i}} \varrho_{2 i} \exp \{2 \varepsilon(s-t)\} x^{T}(s) U_{4} x(s) d s,
\end{aligned}
$$




$$
\begin{aligned}
\Gamma V_{6}( & \left.x_{t}, i\right) \\
= & \dot{x}^{T}(t)\left[\bar{\tau}_{i}^{2} V_{1}+d_{1 i}^{2} V_{2}+\varrho_{1 i}^{2} V_{3}+\varrho_{2 i}^{2} V_{4}\right] \dot{x}(t) \\
& -2 \varepsilon V_{6}\left(x_{t}, i\right) \\
& -\int_{t-\overline{\tau_{i}}}^{t} \overline{\tau_{i}} \exp \{2 \varepsilon(s-t)\} \dot{x}^{T}(s) V_{1} \dot{x}(s) d s \\
& -\int_{t-d_{1 i}}^{t} d_{1 i} \exp \{2 \varepsilon(s-t)\} \dot{x}^{T}(s) V_{2} \dot{x}(s) d s \\
& -\int_{t-d_{m i}}^{t-d_{1 i}} \varrho_{1 i} \exp \{2 \varepsilon(s-t)\} \dot{x}^{T}(s) V_{3} \dot{x}(s) d s \\
& -\int_{t-d_{2 i}}^{t-d_{m i}} \varrho_{2 i} \exp \{2 \varepsilon(s-t)\} \dot{x}^{T}(s) V_{4} \dot{x}(s) d s
\end{aligned}
$$

$$
\begin{aligned}
\Gamma V_{7}\left(x_{t}, i\right) \\
=\dot{x}^{T}(t)\left[\frac{\overline{\tau_{i}}}{4} W_{1}+\frac{d_{1 i}^{4}}{4} W_{2}+\varrho_{3 i}^{2} W_{3}+\varrho_{4 i}^{2} W_{4}\right] \dot{x}(t) \\
-2 \varepsilon V_{7}\left(x_{t}, i\right) \\
\quad-\int_{-\bar{\tau}_{i}}^{0} \int_{t+\theta}^{t} \frac{\bar{\tau}_{i}^{2}}{2} \exp \{2 \varepsilon(s-t)\} \dot{x}^{T}(s) W_{1} \dot{x}(s) d s d \theta \\
-\int_{-d_{1 i}}^{0} \int_{t+\theta}^{t} \frac{d_{1 i}^{2}}{2} \exp \{2 \varepsilon(s-t)\} \dot{x}^{T}(s) W_{2} \dot{x}(s) d s d \theta \\
-\int_{-d_{m i}}^{-d_{1 i}} \int_{t+\theta}^{t} \varrho_{3 i} \exp \{2 \varepsilon(s-t)\} \dot{x}^{T}(s) W_{3} \dot{x}(s) d s d \theta \\
-\int_{-d_{2 i}}^{-d_{m i}} \int_{t+\theta}^{t} \varrho_{4 i} \exp \{2 \varepsilon(s-t)\} \dot{x}^{T}(s) W_{4} \dot{x}(s) d s d \theta .
\end{aligned}
$$

Define

$$
\begin{gathered}
\xi(t)=\operatorname{col}\left\{x(t) \quad \dot{x}(t) \quad x\left(t-d_{i}(t)\right) \quad \dot{x}\left(t-d_{i}(t)\right) \quad x\left(t-d_{1 i}\right) \quad x\left(t-d_{m i}\right) \quad x\left(t-d_{2 i}\right) \quad \dot{x}\left(t-d_{1 i}\right) \quad \dot{x}\left(t-d_{m i}\right) \quad \dot{x}\left(t-d_{2 i}\right)\right. \\
\int_{t-d_{1 i}}^{t} x(s) d s \int_{t-d_{i}(t)}^{t-d_{1 i}} x(s) d s \int_{t-d_{2 i}}^{t-d_{i}(t)} x(s) d s \int_{t-d_{m i}}^{t-d_{1 i}} x(s) d s \int_{t-d_{2 i}}^{t-d_{m i}} x(s) d s \quad x\left(t-\overline{\tau_{i}}\right) \quad \dot{x}\left(t-\overline{\tau_{i}}\right) \\
\left.x\left(t-\tau_{i}(t)\right) \dot{x}\left(t-\tau_{i}(t)\right) \int_{t-\tau_{i}(t)}^{t} x(s) d s \int_{t-\overline{\tau_{i}}}^{t} x(s) d s f_{1} f_{2} f_{3}\right\} .
\end{gathered}
$$

Then, there exist matrices $J=\operatorname{col}\left\{J_{k},(k=1,2, \ldots, 24)\right\}$ with appropriate dimensions, such that the following equality holds according to (13):

$$
\begin{aligned}
2 \xi^{T}(t) J[- & \dot{x}(t)+A_{i} x(t)+B_{i} x\left(t-d_{i}(t)\right) \\
& \left.+C_{i} \dot{x}\left(t-\tau_{i}(t)\right)+D_{i} f_{1}+E_{i} f_{2}+F_{i} f_{3}\right]=0 .
\end{aligned}
$$

Due to $\sum_{j=1}^{N} \pi_{i j}=0$, the following zero equations hold for arbitrary matrices $X_{i}=X_{i}^{T}, Y_{1 i}=Y_{1 i}^{T}, Y_{2 i}=Y_{2 i}^{T}, Z_{1 i}=Z_{1 i}^{T}$, $Z_{2 i}=Z_{2 i}^{T}, i \in S$; that is,

$$
\begin{aligned}
& -x^{T}(t)\left[\sum_{j \in \mathcal{S}_{k}^{i}} \pi_{i j} X_{i}+\sum_{j \in \mathcal{S}_{u k}^{i}} \pi_{i j} X_{i}\right] x(t)=0, \\
& -\int_{t-\bar{\tau}_{j}}^{t} \exp \{2 \varepsilon(s-t)\} x^{T}(s) \\
& \times\left[\sum_{j \in \mathcal{S}_{k}^{i}} \pi_{i j} Y_{1 i}+\sum_{j \in \mathcal{S}_{u k}^{i}} \pi_{i j} Y_{1 i}\right] x(s) d s=0,
\end{aligned}
$$

$$
\begin{aligned}
& -\int_{t-\bar{\tau}_{j}}^{t} \exp \{2 \varepsilon(s-t)\} \dot{x}^{T}(s) \\
& \quad \times\left[\sum_{j \in \mathcal{S}_{k}^{i}} \pi_{i j} Y_{2 i}+\sum_{j \in \mathcal{S}_{u k}^{i}} \pi_{i j} Y_{2 i}\right] \dot{x}(s) d s=0,
\end{aligned}
$$$$
\begin{aligned}
& -\int_{t-d_{2 j}}^{t-d_{1 i}} \exp \{2 \varepsilon(s-t)\} x^{T}(s) \\
& \quad \times\left[\sum_{j \in \mathcal{S}_{k}^{i}} \pi_{i j} Z_{1 i}+\sum_{j \in \mathcal{S}_{u k}^{i}} \pi_{i j} Z_{1 i}\right] x(s) d s=0,
\end{aligned}
$$$$
-\int_{t-d_{2 j}}^{t-d_{1 i}} \exp \{2 \varepsilon(s-t)\} \dot{x}^{T}(s)
$$$$
\times\left[\sum_{j \in \mathcal{S}_{k}^{i}} \pi_{i j} Z_{2 i}+\sum_{j \in \mathcal{S}_{u k}^{i}} \pi_{i j} Z_{2 i}\right] \dot{x}(s) d s=0 .
$$

In view of (9), the following inequalities hold for any scalars $\varepsilon_{1}>0, \varepsilon_{2}>0$, and $\varepsilon_{3}>0$ :

$$
\varepsilon_{1}\left[\alpha^{2} x^{T}(t) x(t)-f_{1}^{T}(x(t), t) f_{1}(x(t), t)\right] \geq 0,
$$




$$
\begin{aligned}
& \varepsilon_{2}[ \beta^{2} x^{T}\left(t-d_{i}(t)\right) x\left(t-d_{i}(t)\right) \\
&-\left.f_{2}^{T}\left(x\left(t-d_{i}(t)\right), t\right) f_{2}\left(x\left(t-d_{i}(t)\right), t\right)\right] \geq 0, \\
& \varepsilon_{3}\left[\gamma^{2} \dot{x}^{T}\left(t-\tau_{i}(t)\right) \dot{x}\left(t-\tau_{i}(t)\right)\right. \\
&\left.\quad-f_{3}^{T}\left(\dot{x}\left(t-\tau_{i}(t)\right), t\right) f_{3}\left(\dot{x}\left(t-\tau_{i}(t)\right), t\right)\right] \geq 0 .
\end{aligned}
$$

From (46) and (61), we have

$$
\begin{aligned}
& \Gamma V(x(t), i, t) \\
& \leq \sum_{k=1}^{7} \Gamma V_{k}\left(x_{t}, i\right) \\
& \quad+\varepsilon_{1}\left[\alpha^{2} x^{T}(t) x(t)-f_{1}^{T} f_{1}\right] \\
& \quad+\varepsilon_{2}\left[\beta^{2} x^{T}\left(t-d_{i}(t)\right) x\left(t-d_{i}(t)\right)-f_{2}^{T} f_{2}\right] \\
& \quad+\varepsilon_{3}\left[\gamma^{2} \dot{x}^{T}\left(t-\tau_{i}(t)\right) \dot{x}\left(t-\tau_{i}(t)\right)-f_{3}^{T} f_{3}\right] .
\end{aligned}
$$

Since it is easy to see $-\left(1-\dot{\tau}_{i}(t)\right) \exp \left\{-2 \varepsilon \tau_{i}(t)\right\} \leq-(1-$ $\left.v_{i}\right) \exp \left\{-2 \varepsilon \overline{\tau_{i}}\right\}$, then from (8) we also obtain that

$$
-\left(1-\dot{d}_{i}(t)\right) \exp \left\{-2 \varepsilon d_{i}(t)\right\} \leq \omega\left(\varepsilon, \mu_{i}\right)
$$

where $\omega\left(\varepsilon, \mu_{i}\right)$ is defined in Theorem 10. Notice (a) of Lemma 7, then

$$
\begin{aligned}
& -\int_{t-d_{1 i}}^{t} d_{1 i} \exp \{2 \varepsilon(s-t)\} x^{T}(s) U_{2} x(s) d s \\
& \leq-\frac{2 \varepsilon d_{1 i}}{\exp \left\{2 \varepsilon d_{1 i}\right\}-1} \xi^{T}(t) e_{11} U_{2} e_{11}^{T} \xi(t) \\
& -\int_{t-d_{1 i}}^{t} d_{1 i} \exp \{2 \varepsilon(s-t)\} \dot{x}^{T}(s) V_{2} \dot{x}(s) d s \\
& \leq-\frac{2 \varepsilon d_{1 i}}{\exp \left\{2 \varepsilon d_{1 i}\right\}-1} \xi^{T}(t)\left(e_{1}-e_{5}\right) V_{2}\left(e_{1}^{T}-e_{5}^{T}\right) \xi(t) .
\end{aligned}
$$

Notice (b) of Lemma 7, then

$$
\begin{gathered}
-\int_{-\bar{\tau}_{i}}^{0} \int_{t+\theta}^{t} \frac{\bar{\tau}_{i}^{2}}{2} \exp \{2 \varepsilon(s-t)\} \dot{x}^{T}(s) W_{1} \dot{x}(s) d s d \theta \\
\leq-\exp \left\{-2 \varepsilon \overline{\tau_{i}}\right\} \xi^{T}(t)\left(\overline{\tau_{i}} e_{1}-e_{21}\right) \\
\quad \times W_{1}\left(\bar{\tau}_{i} e_{1}^{T}-e_{21}^{T}\right) \xi(t)
\end{gathered}
$$

$$
\begin{aligned}
-\int_{-d_{1 i}}^{0} \int_{t+\theta}^{t} \frac{d_{1 i}^{2}}{2} \exp \{2 \varepsilon(s-t)\} \dot{x}^{T}(s) W_{2} \dot{x}(s) d s d \theta \\
\leq-\exp \left\{-2 \varepsilon d_{1 i}\right\} \xi^{T}(t)\left(d_{1 i} e_{1}-e_{11}\right) \\
\quad \times W_{2}\left(d_{1 i} e_{1}^{T}-e_{11}^{T}\right) \xi(t), \\
-\int_{-d_{m i}}^{-d_{1 i}} \int_{t+\theta}^{t} \varrho_{3 i} \exp \{2 \varepsilon(s-t)\} \dot{x}^{T}(s) W_{3} \dot{x}(s) d s d \theta \\
\leq-\exp \left\{-2 \varepsilon d_{m i}\right\} \xi^{T}(t)\left(\varrho_{1 i} e_{1}-e_{14}\right) \\
\quad \times W_{3}\left(\varrho_{1 i} e_{1}^{T}-e_{14}^{T}\right) \xi(t), \\
-\int_{-d_{2 i}}^{-d_{m i}} \int_{t+\theta}^{t} \varrho_{4 i} \exp \{2 \varepsilon(s-t)\} \dot{x}^{T}(s) W_{4} \dot{x}(s) d s d \theta \\
\leq-\exp \left\{-2 \varepsilon d_{2 i}\right\} \xi^{T}(t)\left(\varrho_{2 i} e_{1}-e_{15}\right) \\
\quad \times W_{4}\left(\varrho_{2 i} e_{1}^{T}-e_{15}^{T}\right) \xi(t) .
\end{aligned}
$$

For $d_{i}(t) \in\left[d_{1 i}, d_{m i}\right]$, the following is held from (a) of Lemma 7:

$$
\begin{aligned}
&-\int_{t-d_{m i}}^{t-d_{1 i}} \varrho_{1 i} \exp \{2 \varepsilon(s-t)\} \dot{x}^{T}(s) V_{3} \dot{x}(s) d s \\
&=-\left\{\int_{t-d_{i}(t)}^{t-d_{1 i}}+\int_{t-d_{m i}}^{t-d_{i}(t)}\right\} \varrho_{1 i} \exp \{2 \varepsilon(s-t)\} \\
& \times \dot{x}^{T}(s) V_{3} \dot{x}(s) d s \\
& \leq-\frac{2 \varepsilon \varrho_{1 i}}{\exp \left\{2 \varepsilon d_{i}(t)\right\}-\exp \left\{2 \varepsilon d_{1 i}\right\}} \\
& \times\left[\int_{t-d_{i}(t)}^{t-d_{1 i}} \dot{x}^{T}(s) d s\right] V_{3}\left[\int_{t-d_{i}(t)}^{t-d_{1 i}} \dot{x}(s) d s\right] \\
&-\frac{2 \varepsilon \varrho_{1 i}}{\exp \left\{2 \varepsilon d_{m i}\right\}-\exp \{2 \varepsilon d(t)\}} \\
& \times\left[\int_{t-d_{m i}}^{t-d_{i}(t)} \dot{x}^{T}(s) d s\right] V_{3}\left[\int_{t-d_{m i}}^{t-d_{i}(t)} \dot{x}(s) d s\right] \\
&=-\frac{\left.+\frac{1}{\lambda_{2 i}(t)}\left(e_{3}-e_{6}\right) V_{3}\left(e_{3}^{T}-e_{6}^{T}\right)\right] \xi(t),}{\exp \left\{2 \varepsilon d_{m i}\right\}-\exp \left\{2 \varepsilon d_{1 i}\right\}} \xi^{T}(t) \\
& \times \frac{1}{\lambda_{1 i}(t)}\left(e_{5}-e_{3}\right) V_{3}\left(e_{5}^{T}-e_{3}^{T}\right)
\end{aligned}
$$

where

$$
\begin{aligned}
& \lambda_{1 i}(t)=\frac{\exp \left\{2 \varepsilon d_{i}(t)\right\}-\exp \left\{2 \varepsilon d_{1 i}\right\}}{\exp \left\{2 \varepsilon d_{m i}\right\}-\exp \left\{2 \varepsilon d_{1 i}\right\}} \\
& \lambda_{2 i}(t)=\frac{\exp \left\{2 \varepsilon d_{m i}\right\}-\exp \left\{2 \varepsilon d_{i}(t)\right\}}{\exp \left\{2 \varepsilon d_{m i}\right\}-\exp \left\{2 \varepsilon d_{1 i}\right\}}
\end{aligned}
$$


By Lemma 8, there exists matrix $N_{2}$ with appropriate dimensions such that

$$
\begin{aligned}
& -\int_{t-d_{m i}}^{t-d_{1 i}} \varrho_{1 i} \exp \{2 \varepsilon(s-t)\} \dot{x}^{T}(s) V_{3} \dot{x}(s) d s \\
& \leq-\frac{2 \varepsilon \varrho_{1 i}}{\exp \left\{2 \varepsilon d_{m i}\right\}-\exp \left\{2 \varepsilon d_{1 i}\right\}} \xi^{T}(t) \\
& \times\left[e_{5}-e_{3} e_{3}-e_{6}\right]\left[\begin{array}{cc}
V_{3} & N_{2} \\
N_{2}^{T} & V_{3}
\end{array}\right]\left[\begin{array}{c}
e_{5}^{T}-e_{3}^{T} \\
e_{3}^{T}-e_{6}^{T}
\end{array}\right] \xi(t), \\
& {\left[\begin{array}{cc}
V_{3} & N_{2} \\
N_{2}^{T} & V_{3}
\end{array}\right]>0 .}
\end{aligned}
$$

Similarly, considering $-\int_{t-d_{m i}}^{t-d_{1 i}} \varrho_{1 i} \exp \{2 \varepsilon(s-t)\} x^{T}(s) U_{3} x$ $(s) d s$ and following the same procedure, there exists matrix $N_{1}$ with appropriate dimensions such that

$$
\begin{aligned}
& -\int_{t-d_{m i}}^{t-d_{1 i}} \varrho_{1 i} \exp \{2 \varepsilon(s-t)\} x^{T}(s) U_{3} x(s) d s \\
& \leq-\frac{2 \varepsilon \varrho_{1 i}}{\exp \left\{2 \varepsilon d_{m i}\right\}-\exp \left\{2 \varepsilon d_{1 i}\right\}} \xi^{T}(t) \\
& \times\left[\begin{array}{ll}
e_{12} & e_{14}-e_{12}
\end{array}\right]\left[\begin{array}{cc}
U_{3} & N_{1} \\
N_{1}^{T} & U_{3}
\end{array}\right]\left[\begin{array}{c}
e_{12}^{T} \\
e_{14}^{T}-e_{12}^{T}
\end{array}\right] \xi(t), \\
& {\left[\begin{array}{cc}
U_{3} & N_{1} \\
N_{1}^{T} & U_{3}
\end{array}\right]>0 .}
\end{aligned}
$$

For $\tau_{i}(t) \in\left[0, \bar{\tau}_{i}\right]$, with the same matrix inequalities technique, we obtain the following:

$$
\begin{aligned}
& -\int_{t-\overline{\tau_{i}}}^{t} \overline{\tau_{i}} \exp \{2 \varepsilon(s-t)\} x^{T}(s) U_{1} x(s) d s \\
& \leq-\frac{2 \varepsilon \overline{\tau_{i}}}{\exp \left\{2 \varepsilon \overline{\tau_{i}}\right\}-1} \xi^{T}(t)\left[\begin{array}{ll}
e_{20} & e_{21}-e_{20}
\end{array}\right] \\
& \times\left[\begin{array}{cc}
U_{1} & M_{1} \\
M_{1}^{T} & U_{1}
\end{array}\right]\left[\begin{array}{c}
e_{20}^{T} \\
e_{21}^{T}-e_{20}^{T}
\end{array}\right] \xi(t), \\
& {\left[\begin{array}{ll}
U_{1} & M_{1} \\
M_{1}^{T} & U_{1}
\end{array}\right]>0} \\
& -\int_{t-\overline{\tau_{i}}}^{t} \overline{\tau_{i}} \exp \{2 \varepsilon(s-t)\} \dot{x}^{T}(s) V_{1} \dot{x}(s) d s \\
& \leq-\frac{2 \varepsilon \overline{\tau_{i}}}{\exp \left\{2 \varepsilon \overline{\tau_{i}}\right\}-1} \xi^{T}(t)\left[e_{1}-e_{18} \quad e_{18}-e_{16}\right] \\
& \times\left[\begin{array}{cc}
V_{1} & M_{2} \\
M_{2}^{T} & V_{1}
\end{array}\right]\left[\begin{array}{c}
e_{1}^{T}-e_{18}^{T} \\
e_{18}^{T}-e_{16}^{T}
\end{array}\right] \xi(t), \\
& {\left[\begin{array}{cc}
V_{1} & M_{2} \\
M_{2}^{T} & V_{1}
\end{array}\right]>0}
\end{aligned}
$$

Consider $-\int_{t-d_{2 i}}^{t-d_{m i}} \varrho_{2 i} \exp \{2 \varepsilon(s-t)\} x^{T}(s) U_{4} x(s) d s$ and $-\int_{t-d_{2 i}}^{t-d_{m i}} \varrho_{2 i} \exp \{2 \varepsilon(s-t)\} \dot{x}^{T}(s) V_{4} \dot{x}(s) d s$, which are directly estimated by (a) of Lemma 7; that is,

$$
\begin{aligned}
& -\int_{t-d_{2 i}}^{t-d_{m i}} \varrho_{2 i} \exp \{2 \varepsilon(s-t)\} x^{T}(s) U_{4} x(s) d s \\
& \leq-\frac{2 \varepsilon \varrho_{2 i}}{\exp \left\{2 \varepsilon d_{2 i}\right\}-\exp \left\{2 \varepsilon d_{m i}\right\}} \xi^{T}(t) e_{15} U_{4} e_{15}^{T} \xi(t), \\
& -\int_{t-d_{2 i}}^{t-d_{m i}} \varrho_{2 i} \exp \{2 \varepsilon(s-t)\} \dot{x}^{T}(s) V_{4} \dot{x}(s) d s \\
& \leq-\frac{2 \varepsilon \varrho_{2 i}}{\exp \left\{2 \varepsilon d_{2 i}\right\}-\exp \left\{2 \varepsilon d_{m i}\right\}} \xi^{T}(t) \\
& \quad \times\left(e_{6}-e_{7}\right) V_{4}\left(e_{6}^{T}-e_{7}^{T}\right) \xi(t) .
\end{aligned}
$$

Substituting (47), (54)-(60), and (63)-(71) into (62) we obtain

$$
\begin{aligned}
& \Gamma V(x(t), i, t)+2 \varepsilon V(x(t), i, t) \\
& \quad \leq \xi^{T}(t)\left(\Omega_{i 0}^{\varepsilon}+\Omega_{i 1}^{\varepsilon}\right) \xi(t)+\mho,
\end{aligned}
$$

where

$$
\begin{aligned}
& \mho=\int_{t-\overline{\tau_{j}}}^{t} \exp \{2 \varepsilon(s-t)\} x^{T}(s)\left\{\sum_{j \neq i} \pi_{i j} Q_{1 j}-\sum_{j \in \mathcal{S}_{k}^{i}} \pi_{i j} Y_{1 i}\right. \\
& \left.-\sum_{j \in \mathcal{S}_{u k}^{i}} \pi_{i j} Y_{1 i}\right\} x(s) d s \\
& +\int_{t-\overline{\tau_{j}}}^{t} \exp \{2 \varepsilon(s-t)\} \dot{x}^{T}(s)\left\{\sum_{j \neq i} \pi_{i j} Q_{2 j}-\sum_{j \in \mathcal{S}_{k}^{i}} \pi_{i j} Y_{2 i}\right. \\
& \left.-\sum_{j \in \mathcal{S}_{u k}^{i}} \pi_{i j} Y_{2 i}\right\} \dot{x}(s) d s \\
& +\int_{t-d_{2 j}}^{t-d_{1 i}} \exp \{2 \varepsilon(s-t)\} x^{T}(s)\left\{\sum_{j \neq i} \pi_{i j} R_{1 j}-\sum_{j \in \mathcal{S}_{k}^{i}} \pi_{i j} Z_{1 i}\right. \\
& \left.-\sum_{j \in \mathcal{S}_{u k}^{i}} \pi_{i j} Z_{1 i}\right\} x(s) d s \\
& +\int_{t-d_{2 j}}^{t-d_{1 i}} \exp \{2 \varepsilon(s-t)\} \dot{x}^{T}(s)\left\{\sum_{j \neq i} \pi_{i j} T_{1 j}-\sum_{j \in \delta_{k}^{i}} \pi_{i j} Z_{2 i}\right. \\
& \left.-\sum_{j \in \delta_{u k}^{i}} \pi_{i j} Z_{2 i}\right\} x(s) d s \\
& +x^{T}(t)\left\{\sum_{j \in \mathcal{S}_{u k}^{i}} \pi_{i j}\left(P_{j}-X_{i}\right)\right\} x(t) .
\end{aligned}
$$



terms

On the other hand, for $d_{i}(t) \in\left[d_{m i}, d_{2 i}\right]$, the integral

$$
\begin{aligned}
& -\int_{t-d_{2 i}}^{t-d_{m i}} \varrho_{2 i} \exp \{2 \varepsilon(s-t)\} x^{T}(s) U_{4} x(s) d s, \\
& -\int_{t-d_{2 i}}^{t-d_{m i}} \varrho_{2 i} \exp \{2 \varepsilon(s-t)\} \dot{x}^{T}(s) V_{4} \dot{x}(s) d s
\end{aligned}
$$

are disposed and estimated by Lemma 8 , and

$$
\begin{aligned}
& -\int_{t-d_{m i}}^{t-d_{1 i}} \varrho_{1 i} \exp \{2 \varepsilon(s-t)\} x^{T}(s) U_{3} x(s) d s, \\
& -\int_{t-d_{m i}}^{t-d_{1 i}} \varrho_{1 i} \exp \{2 \varepsilon(s-t)\} \dot{x}^{T}(s) V_{3} \dot{x}(s) d s
\end{aligned}
$$

are directly estimated by (a) of Lemma 7. Therefore,

$$
\begin{aligned}
& \Gamma V(x(t), i, t)+2 \varepsilon V(x(t), i, t) \\
& \quad \leq \xi^{T}(t)\left(\Omega_{i 0}^{\varepsilon}+\Omega_{i 2}^{\varepsilon}\right) \xi(t)+\mho .
\end{aligned}
$$

With (72) and (76), the following inequality (77) is held for $d_{i}(t) \in\left[d_{1 i}, d_{2 i}\right]$ if (26)-(34) are satisfied

$$
\Gamma V(x(t), i, t)+2 \varepsilon V(x(t), i, t)<0 .
$$

From the stochastic Lyapunov functional (38) and (77), it is held that

$$
\begin{aligned}
V(x(t), i, t) \geq & \min _{i \in S}\left\{\lambda_{\min }\left(P_{i}\right)\right\}\|x(t)\|^{2} \\
= & \underline{\lambda}\|x(t)\|^{2}, \\
V(x(t), i, t)< & \exp \left\{-2 \varepsilon\left(t-t_{0}\right)\right\} \\
& \times V\left(x_{t_{0}}, r_{t_{0}}, t_{0}\right) .
\end{aligned}
$$

Moreover, we have

$$
V\left(x_{t_{0}}, r_{t_{0}}, t_{0}\right)=\sum_{k=1}^{6} V_{k}\left(x_{t_{0}}, r_{t_{0}}\right) \leq \bar{\lambda}\left\|x_{t_{0}}\right\|_{\zeta}^{2}
$$

Then from (78) and (79), it is readily seen that

$$
\|x(t)\| \leq \sqrt{\frac{\bar{\lambda}}{\underline{\lambda}}} \exp \left\{-\varepsilon\left(t-t_{0}\right)\right\}\left\|x_{t_{0}}\right\|_{\zeta^{\prime}}
$$

where $\kappa=\sqrt{\bar{\lambda} / \underline{\lambda}} \geq 1$.

Therefore, by Definition 4, the system (13) is exponentially stable with a decay rate $\varepsilon$. This completes the proof.
Remark 12. It is noted that the integral intervals in (79) are enlarged as follows:

$$
\begin{aligned}
\sum_{k=1}^{6} V_{k}\left(x_{t_{0}}, r_{t_{0}}\right) \\
\leq \int_{t_{0}-\varsigma}^{t_{0}} \exp \left\{2 \varepsilon\left(s-t_{0}\right)\right\} x^{T}(s) \\
\quad \times\left\{Q_{1 i}+Q_{3}+R_{1 i}+\sum_{k=2}^{4} R_{k}\right\} x(s) d s \\
+\int_{t_{0}-\varsigma}^{t_{0}} \exp \left\{2 \varepsilon\left(s-t_{0}\right)\right\} \dot{x}^{T}(s) \\
\quad \times\left\{Q_{2 i}+Q_{4}+T_{1 i}+\sum_{l=2}^{4} T_{l}\right\} \dot{x}(s) d s \\
+\int_{-\varsigma}^{0} \int_{t_{0}+\theta}^{t_{0}} \varsigma \exp \left\{2 \varepsilon\left(s-t_{0}\right)\right\} x^{T}(s) \\
\quad \times\left(\sum_{m=1}^{4} U_{m}\right) x(s) d s d \theta \\
\quad+\int_{-\varsigma}^{0} \int_{t_{0}+\theta}^{t_{0}} \varsigma \exp \left\{2 \varepsilon\left(s-t_{0}\right)\right\} \dot{x}^{T}(s) \\
\quad \times\left(\sum_{n=1}^{4} V_{n}\right) \dot{x}(s) d s d \theta \\
+\int_{-\varsigma}^{0} \int_{\theta}^{0} \int_{t_{0}+\lambda}^{t_{0}} \frac{\varsigma^{2}}{2} \exp \left\{2 \varepsilon\left(s-t_{0}\right)\right\} \dot{x}^{T}(s)
\end{aligned}
$$

(79) can be obtained by letting $\bar{\lambda}$ be defined as previously mentioned.

Remark 13. In Theorem 10, the factors

$$
\begin{aligned}
& \frac{\exp \left\{2 \varepsilon d_{2 i}\right\}-\exp \left\{2 \varepsilon d_{i}(t)\right\}}{2 \varepsilon}, \\
& \frac{\exp \left\{2 \varepsilon d_{i}(t)\right\}-\exp \left\{2 \varepsilon d_{1 i}\right\}}{2 \varepsilon}
\end{aligned}
$$

may be enlarged as $\left(\exp \left\{2 \varepsilon d_{2 i}\right\}-\exp \left\{2 \varepsilon d_{1 i}\right\}\right) / 2 \varepsilon$. This will lead conservative results due to the fact that $d_{i}(t)$ cannot achieve $d_{1 i}$ and $d_{2 i}$ at the same time. While we apply Lemma 7 to these terms, the method by using reciprocally convex lemma [38] can achieve less conservative results. Moreover, for $\varepsilon>0$, the factor $\left(\dot{d}_{i}(t)-1\right) \exp \left\{-2 \varepsilon d_{i}(t)\right\}$ that appeared in the derivative of Lyapunov functional may be directly enlarged as $\mu_{i} \exp \left\{-2 \varepsilon d_{1 i}\right\}-\exp \left\{-2 \varepsilon d_{2 i}\right\}$. In this paper, we 
enlarge it as $\omega\left(\varepsilon, \mu_{i}\right)$ to reduce the conservativeness of the obtained criteria. In the literature $[36,45,46]$, this factor is enlarged as $\left(\mu_{i}-1\right) \exp \left\{-2 \varepsilon d_{2 i}\right\}$, which only holds for $\mu_{i}<1$.

Remark 14. The information on the lower bound of the delay is sufficiently used in the Lyapunov functional by introducing the terms such as $\int_{t-d_{m i}}^{t-d_{1 i}} \exp \{2 \varepsilon(s-t)\} x^{T}(s) R_{3} x(s) d s$, $\int_{t-d_{2 i}}^{t-d_{m i}} \exp \{2 \varepsilon(s-t)\} x^{T}(s) R_{4} x(s) d s$, and $\int_{t-d_{i}(t)}^{t-d_{1 i}} \exp \{2 \varepsilon(s-$ t) $\} x^{T}(s) R_{1 i} x(s) d s$, which is equivalent to the improved bounding technique.

Remark 15. It should be also mentioned that the result obtained in Theorem 10 is delay-range-dependent and decay rate-dependent stability condition for (13), which is less conservative than the previous ones and will be verified in Section 4. Although the large number of introduced free weighting matrices may increase the complexity of computation, utilizing the technique of free weighting matrices would reduce the conservativeness. In addition, the given results can be extended to more general systems with neutral delay $\tau_{i}(t)$. That is, $\tau_{1 i} \leq \tau_{i}(t) \leq \tau_{2 i}$. The corresponding results can be obtained by using the similar methods.

In Theorem 10, it is assumed that $\varepsilon \neq 0$. For $\varepsilon=0$, by L'Hospital rule, the following asymptotic stability criterion can be obtained.

Corollary 16. For given scalars $\pi_{i}^{m}, \pi_{i}^{M}, \alpha, \beta, \gamma, \overline{\tau_{i}}, v_{i}, d_{1 i}$, $d_{2 i}, \mu_{i}$ and constant scalar $d_{m i}$ satisfying $d_{1 i}<d_{m i}<d_{2 i}$, the systems described by (13) with partially known transition rates are asymptotically stable if $\left\|C_{i}\right\|+\gamma<1$ and there exist symmetric positive matrices $P_{i}>0, Q_{1 i}>0, Q_{2 i}>0, R_{1 i}>0$, $T_{1 i}>0,(i \in S), Q_{j}>0,(j=3,4), R_{k}>0, T_{l}>0$, $(k, l=2,3,4), U_{m}>0, V_{n}>0, W_{s}>0,(m, n, s=1,2,3,4)$ and matrices $M_{1}, M_{2}, N_{1}, N_{2}, N_{3}, N_{4}$ for any scalars $\varepsilon_{1}, \varepsilon_{2}, \varepsilon_{3}$, any symmetric matrices $X_{i}, Y_{1 i}, Y_{2 i}, Z_{1 i}, Z_{2 i}$, $(i \in S)$ and any matrices $J_{k},(k=1,2, \ldots, 24)$ with appropriate dimensions, such that (26)-(32) and the following linear matrix inequalities hold:

$$
\begin{aligned}
& \text { (i) }\left[\begin{array}{ll}
U_{3} & N_{1} \\
N_{1}^{T} & U_{3}
\end{array}\right]>0, \quad\left[\begin{array}{cc}
V_{3} & N_{2} \\
N_{2}^{T} & V_{3}
\end{array}\right]>0, \\
& \text { (ii) } \Omega_{i 0}+\Omega_{i 1}<0, \\
& \text { (iii) }\left[\begin{array}{ll}
U_{4} & N_{3} \\
N_{3}^{T} & U_{4}
\end{array}\right]>0, \quad\left[\begin{array}{cc}
V_{4} & N_{4} \\
N_{4}^{T} & V_{4}
\end{array}\right]>0,
\end{aligned}
$$

(iv) $\Omega_{i 0}+\Omega_{i 2}<0$,

where

$$
\begin{aligned}
\Omega_{i 0}= & \sum_{m=1}^{24} e_{m} \widehat{\Upsilon}_{m} e_{m}^{T}+\mathscr{L}(\Xi) \\
& -\left(e_{1}-e_{5}\right) V_{2}\left(e_{1}^{T}-e_{5}^{T}\right) \\
& -\left(\bar{\tau}_{i} e_{1}-e_{21}\right) W_{1}\left(\bar{\tau}_{i} e_{1}^{T}-e_{21}^{T}\right) \\
& -\left(d_{1 i} e_{1}-e_{11}\right) W_{2}\left(d_{1 i} e_{1}^{T}-e_{11}^{T}\right)
\end{aligned}
$$

$$
\begin{aligned}
& -\left(\varrho_{1 i} e_{1}-e_{14}\right) W_{3}\left(\varrho_{1 i} e_{1}^{T}-e_{14}^{T}\right) \\
& -\left(\varrho_{2 i} e_{1}-e_{15}\right) W_{4}\left(\varrho_{2 i} e_{1}^{T}-e_{15}^{T}\right) \\
& -\left[\begin{array}{ll}
e_{20} & e_{21}-e_{20}
\end{array}\right]\left[\begin{array}{cc}
U_{1} & M_{1} \\
M_{1}^{T} & U_{1}
\end{array}\right]\left[\begin{array}{c}
e_{20}^{T} \\
e_{21}^{T}-e_{20}^{T}
\end{array}\right] \\
& -\left[e_{1}-e_{18} e_{18}-e_{16}\right]\left[\begin{array}{cc}
V_{1} & M_{2} \\
M_{2}^{T} & V_{1}
\end{array}\right]\left[\begin{array}{c}
e_{1}^{T}-e_{18}^{T} \\
e_{18}^{T}-e_{16}^{T}
\end{array}\right] \text {, } \\
& \widehat{\Upsilon}_{1}=A_{i}^{T} P_{i}+P_{i} A_{i}+\sum_{j \in \mathcal{S}_{k}^{i}} \pi_{i j}\left(P_{j}-X_{i}\right) \\
& +Q_{1 i}+Q_{3}+R_{2}+\bar{\tau}_{i}^{2} U_{1}+d_{1 i}^{2} U_{2} \\
& +\varrho_{1 i}^{2} U_{3}+\varrho_{2 i}^{2} U_{4}+\varepsilon_{1} \alpha^{2} I+J_{1} A_{i}+A_{i}^{T} J_{1}^{T}, \\
& \widehat{\Upsilon}_{3}=-\left(1-\mu_{i}\right) R_{1 i}+\varepsilon_{2} \beta^{2} I+J_{3} B_{i}+B_{i}^{T} J_{3}^{T} \text {, } \\
& \widehat{\Upsilon}_{4}=-\left(1-\mu_{i}\right) T_{1 i} \text {, } \\
& \widehat{\Upsilon}_{5}=R_{1 i}+R_{3}-R_{2}, \quad \widehat{\Upsilon}_{6}=R_{4}-R_{3} \text {, } \\
& \widehat{\Upsilon}_{7}=-R_{4}, \quad \hat{\Upsilon}_{8}=T_{1 i}+T_{3}-T_{2}, \\
& \widehat{\Upsilon}_{9}=T_{4}-T_{3}, \quad \widehat{\Upsilon}_{10}=-T_{4}, \quad \widehat{\Upsilon}_{11}=-U_{2}, \\
& \widehat{\Upsilon}_{16}=-Q_{3}, \quad \widehat{\Upsilon}_{17}=-Q_{4}, \quad \widehat{\Upsilon}_{18}=-\left(1-v_{i}\right) Q_{1 i} \text {, } \\
& \widehat{\Upsilon}_{19}=-\left(1-v_{i}\right) Q_{2 i}+\varepsilon_{3} \gamma^{2} I+J_{19} C_{i}+C_{i}^{T} J_{19}^{T} \text {. }
\end{aligned}
$$

Other $\widehat{\Upsilon}_{m}=\Upsilon_{m},(m=2,12,13,14,15,20, \ldots, 24)$, which have been defined in Theorem 10,

$$
\begin{aligned}
\Omega_{i 1}= & -e_{15} U_{4} e_{15}^{T}-\left(e_{6}-e_{7}\right) V_{4}\left(e_{6}^{T}-e_{7}^{T}\right) \\
& -\left[\begin{array}{ll}
e_{12} & e_{14}-e_{12}
\end{array}\right]\left[\begin{array}{ll}
U_{3} & N_{1} \\
N_{1}^{T} & U_{3}
\end{array}\right]\left[\begin{array}{c}
e_{12}^{T} \\
e_{14}^{T}-e_{12}^{T}
\end{array}\right] \\
& -\left[\begin{array}{ll}
e_{5}-e_{3} & e_{3}-e_{6}
\end{array}\right]\left[\begin{array}{cc}
V_{3} & N_{2} \\
N_{2}^{T} & V_{3}
\end{array}\right]\left[\begin{array}{c}
e_{5}^{T}-e_{3}^{T} \\
e_{3}^{T}-e_{6}^{T}
\end{array}\right], \\
\Omega_{i 2}= & -e_{14} U_{3} e_{14}^{T}-\left(e_{5}-e_{6}\right) V_{3}\left(e_{5}^{T}-e_{6}^{T}\right) \\
& -\left[\begin{array}{lll}
e_{13} & e_{15}-e_{13}
\end{array}\right]\left[\begin{array}{ll}
U_{4} & N_{3} \\
N_{3}^{T} & U_{4}
\end{array}\right]\left[\begin{array}{c}
e_{13}^{T} \\
e_{15}^{T}-e_{13}^{T}
\end{array}\right] \\
& -\left[\begin{array}{ll}
e_{3}-e_{7} & e_{6}-e_{3}
\end{array}\right]\left[\begin{array}{ll}
V_{4} & N_{4} \\
N_{4}^{T} & V_{4}
\end{array}\right]\left[\begin{array}{c}
e_{3}^{T}-e_{7}^{T} \\
e_{6}^{T}-e_{3}^{T}
\end{array}\right]
\end{aligned}
$$

and the remaining notations are the same as Theorem 10.

Further, the information on the transition rates may be completely unknown in some circumstances, which viewed the systems as switched systems with arbitrary switching. The following corollary is therefore given to guarantee the exponential stability for this case.

Corollary 17. For given scalars $\pi_{i}^{m}, \pi_{i}^{M}, \alpha, \beta, \gamma, \varepsilon, \overline{\tau_{i}}, v_{i}, d_{1 i}$, $d_{2 i}, \mu_{i}$, and constant scalar $d_{m i}$ satisfying $d_{1 i}<d_{m i}<d_{2 i}$, the 
systems described by (13) with completely unknown transition rates are exponentially stable with decay rate $\varepsilon$ and $\kappa=\sqrt{\bar{\lambda} / \lambda}$ if $\left\|C_{i}\right\|+\gamma<1$ and there exist symmetric positive matrices $P>0, Q_{j}>0, R_{k}>0, T_{l}>0, U_{m}>0, V_{n}>0, W_{s}>0$, $(j, k, l, m, n, s=1,2,3,4)$ and matrices $M_{1}, M_{2}, N_{1}, N_{2}, N_{3}$, $N_{4}$ for any scalars $\varepsilon_{1}, \varepsilon_{2}, \varepsilon_{3}$, any symmetric matrices $Y_{1}, Y_{2}, Z_{1}$, $Z_{2}$, and any matrices $J_{k},(k=1,2, \ldots, 24)$ with appropriate dimensions, such that the following linear matrix inequalities hold:

$$
\begin{gathered}
-\sum_{j \in \mathcal{S}_{u k}^{i}, j \neq i} \pi_{i}^{M}\left(Q_{1}-Y_{1}\right)-\pi_{i}^{m} Y_{1} \leq 0 ; \\
-\sum_{j \in \mathcal{S}_{u k, j}^{i}, j \neq i} \pi_{i}^{M}\left(Q_{2}-Y_{2}\right)-\pi_{i}^{m} Y_{2} \leq 0, \\
-\sum_{j \in \mathcal{S}_{u k}^{i}, j \neq i} \pi_{i}^{M}\left(R_{1}-Z_{1}\right)-\pi_{i}^{m} Z_{1} \leq 0 ; \\
-\sum_{j \in \mathcal{S}_{u k}^{i}, j \neq i} \pi_{i}^{M}\left(T_{1}-Z_{2}\right)-\pi_{i}^{m} Z_{2} \leq 0, \\
\text { (i) }\left[\begin{array}{cc}
U_{3} & N_{1} \\
N_{1}^{T} & U_{3}
\end{array}\right]>0, \quad\left[\begin{array}{cc}
V_{3} & N_{2} \\
N_{2}^{T} & V_{3}
\end{array}\right]>0,
\end{gathered}
$$$$
\text { (ii) } \widetilde{\Omega}_{i 0}^{\varepsilon}+\widetilde{\Omega}_{i 1}^{\varepsilon}<0,
$$$$
\text { (iii) }\left[\begin{array}{cc}
U_{4} & N_{3} \\
N_{3}^{T} & U_{4}
\end{array}\right]>0, \quad\left[\begin{array}{cc}
V_{4} & N_{4} \\
N_{4}^{T} & V_{4}
\end{array}\right]>0,
$$

(iv) $\widetilde{\Omega}_{i 0}^{\varepsilon}+\widetilde{\Omega}_{i 2}^{\varepsilon}<0$,

where

$$
\begin{aligned}
\widetilde{\Omega}_{i 0}^{\varepsilon}= & \sum_{m=1}^{24} e_{m} \widetilde{\Upsilon}_{m} e_{m}^{T}+\mathscr{L}(\Xi) \\
& -\frac{2 \varepsilon d_{1 i}}{\exp \left\{2 \varepsilon d_{1 i}\right\}-1}\left(e_{1}-e_{5}\right) V_{2}\left(e_{1}^{T}-e_{5}^{T}\right) \\
& -\exp \left\{-2 \varepsilon \overline{\tau_{i}}\right\}\left(\bar{\tau}_{i} e_{1}-e_{21}\right) W_{1}\left(\bar{\tau}_{i} e_{1}^{T}-e_{21}^{T}\right) \\
& -\exp \left\{-2 \varepsilon d_{1 i}\right\}\left(d_{1 i} e_{1}-e_{11}\right) W_{2}\left(d_{1 i} e_{1}^{T}-e_{11}^{T}\right) \\
& -\exp \left\{-2 \varepsilon d_{m i}\right\}\left(\varrho_{1 i} e_{1}-e_{14}\right) W_{3}\left(\varrho_{1 i} e_{1}^{T}-e_{14}^{T}\right) \\
& -\exp \left\{-2 \varepsilon d_{2 i}\right\}\left(\varrho_{2 i} e_{1}-e_{15}\right) W_{4}\left(\varrho_{2 i} e_{1}^{T}-e_{15}^{T}\right) \\
& -\frac{2 \varepsilon \overline{\tau_{i}}}{\exp \left\{2 \varepsilon \overline{\tau_{i}}\right\}-1}\left[e_{20} e_{21}-e_{20}\right] \\
& \times\left[\begin{array}{cc}
U_{1} & M_{1} \\
M_{1}^{T} & U_{1}
\end{array}\right]\left[\begin{array}{c}
e_{20}^{T} \\
e_{21}^{T}-e_{20}^{T}
\end{array}\right] \\
& -\frac{2 \varepsilon \overline{\tau_{i}}}{\exp \left\{2 \varepsilon \overline{\tau_{i}}\right\}-1}\left[e_{1}-e_{18} e_{18}-e_{16}\right] \\
& \times\left[\begin{array}{cc}
V_{1} & M_{2} \\
M_{2}^{T} & V_{1}
\end{array}\right]\left[\begin{array}{l}
e_{1}^{T}-e_{18}^{T} \\
e_{18}^{T}-e_{16}^{T}
\end{array}\right], \\
& \widetilde{\Upsilon}_{1}=A_{i}^{T} P+P A_{i}+2 \varepsilon P+Q_{1}+Q_{3} \\
& +R_{2}+\bar{\tau}_{i}^{2} U_{1}+d_{1 i}^{2} U_{2}+\varrho_{1 i}^{2} U_{3} \\
& +\varrho_{2 i}^{2} U_{4}+\varepsilon_{1} \alpha^{2} I+J_{1} A_{i}+A_{i}^{T} J_{1}^{T},
\end{aligned}
$$$$
\tilde{\Upsilon}_{2}=Q_{2}+Q_{4}+T_{2}+\bar{\tau}_{i}^{2} V_{1}+d_{1 i}^{2} V_{2}
$$$$
+\varrho_{1 i}^{2} V_{3}+\varrho_{2 i}^{2} V_{4}+\frac{\overline{\tau_{i}^{4}}}{4} W_{1}+\frac{d_{1 i}^{4}}{4} W_{2}
$$$$
+\varrho_{3 i}^{2} W_{3}+\varrho_{4 i}^{2} W_{4}-J_{2}-J_{2}^{T} \text {, }
$$$$
\widetilde{\Upsilon}_{3}=\omega\left(\varepsilon, \mu_{i}\right) R_{1}+\varepsilon_{2} \beta^{2} I+J_{3} B_{i}+B_{i}^{T} J_{3}^{T} \text {, }
$$$$
\widetilde{\Upsilon}_{4}=\varpi\left(\varepsilon, \mu_{i}\right) T_{1} \text {, }
$$$$
\widetilde{\Upsilon}_{5}=\exp \left\{-2 \varepsilon d_{1 i}\right\}\left(R_{1}+R_{3}-R_{2}\right) \text {, }
$$$$
\tilde{\Upsilon}_{6}=\exp \left\{-2 \varepsilon d_{m i}\right\}\left(R_{4}-R_{3}\right),
$$$$
\widetilde{\Upsilon}_{7}=-\exp \left\{-2 \varepsilon d_{2 i}\right\} R_{4} \text {, }
$$$$
\widetilde{Y}_{8}=\exp \left\{-2 \varepsilon d_{1 i}\right\}\left(T_{1}+T_{3}-T_{2}\right) \text {, }
$$$$
\widetilde{\Upsilon}_{9}=\exp \left\{-2 \varepsilon d_{m i}\right\}\left(T_{4}-T_{3}\right) \text {, }
$$$$
\widetilde{\Upsilon}_{10}=-\exp \left\{-2 \varepsilon d_{2 i}\right\} T_{4},
$$$$
\widetilde{\Upsilon}_{11}=-\frac{2 \varepsilon d_{1 i}}{\exp \left\{2 \varepsilon d_{1 i}\right\}-1} U_{2},
$$$$
\widetilde{\Upsilon}_{16}=-\exp \left\{-2 \varepsilon \bar{\varepsilon} \bar{\tau}_{i}\right\} Q_{3} \text {, }
$$$$
\widetilde{\Upsilon}_{17}=-\exp \left\{-2 \varepsilon \bar{\varepsilon} \bar{\tau}_{i}\right\} Q_{4} \text {, }
$$$$
\widetilde{\Upsilon}_{18}=-\left(1-v_{i}\right) \exp \left\{-2 \varepsilon \bar{\varepsilon} \bar{\tau}_{i}\right\} Q_{1},
$$

$$
\begin{gathered}
\tilde{\Upsilon}_{19}=-\left(1-v_{i}\right) \exp \left\{-2 \varepsilon \bar{\tau}_{i}\right\} Q_{2}+\varepsilon_{3} \gamma^{2} I+J_{19} C_{i}+C_{i}^{T} J_{19}^{T}, \\
\tilde{\Upsilon}_{22}=-\varepsilon_{1} I+J_{22} D_{i}+D_{i}^{T} J_{22}^{T}, \\
\tilde{\Upsilon}_{23}=-\varepsilon_{2} I+J_{23} E_{i}+E_{i}^{T} J_{23}^{T}, \\
\tilde{\Upsilon}_{24}=-\varepsilon_{3} I+J_{24} F_{i}+F_{i}^{T} J_{24}^{T}, \\
\widetilde{\Upsilon}_{m}=0, \quad(m=12,13,14,15,20,21),
\end{gathered}
$$

where

$$
\begin{aligned}
& \widetilde{\Omega}_{i 1}^{\varepsilon}=\Omega_{i 1}^{\varepsilon}, \quad \widetilde{\Omega}_{i 2}^{\varepsilon}=\Omega_{i 2}^{\varepsilon}, \\
& \bar{\lambda}= \lambda_{\max }(P)+\frac{1-\exp \{-2 \varepsilon \varsigma\}}{2 \varepsilon} \\
& \times\left\{\sum_{j=1}^{4} \lambda_{\max }\left(Q_{j}\right)+\sum_{k=1}^{4} \lambda_{\max }\left(R_{k}\right)+\sum_{l=1}^{4} \lambda_{\max }\left(T_{l}\right)\right\} \\
&+\frac{\exp \{-2 \varepsilon \zeta\}+2 \varepsilon \varsigma-1}{4 \varepsilon^{2}} \\
& \times\left\{\sum_{m=1}^{4} \lambda_{\max }\left(U_{m}\right)+\sum_{n=1}^{4} \lambda_{\max }\left(V_{n}\right)\right\} \\
&+\frac{1-\exp \{-2 \varepsilon \varsigma\}+2 \varepsilon^{2} \varsigma^{2}-2 \varepsilon \varsigma}{8 \varepsilon^{3}}\left\{\sum_{s=1}^{4} \lambda_{\max }\left(W_{s}\right)\right\}
\end{aligned}
$$

and the remaining notations are the same as Theorem 10. 
Proof. Since the elements of transition rates are entirely unknown, we choose the Lyapunov functional as follows:

$$
V(x(t), i, t)=x^{T}(t) P x(t)+\sum_{k=2}^{7} V_{k}\left(x_{t}, i\right),
$$

where

$$
\begin{aligned}
& V_{2}\left(x_{t}, i\right)=\int_{t-\tau_{i}(t)}^{t} \exp \{2 \varepsilon(s-t)\} x^{T}(s) Q_{1} x(s) d s \\
& +\int_{t-\tau_{i}(t)}^{t} \exp \{2 \varepsilon(s-t)\} \dot{x}^{T}(s) Q_{2} \dot{x}(s) d s \\
& +\int_{t-\overline{\tau_{i}}}^{t} \exp \{2 \varepsilon(s-t)\} x^{T}(s) Q_{3} x(s) d s \\
& +\int_{t-\overline{\tau_{i}}}^{t} \exp \{2 \varepsilon(s-t)\} \dot{x}^{T}(s) Q_{4} \dot{x}(s) d s \\
& V_{3}\left(x_{t}, i\right)=\int_{t-d_{i}(t)}^{t-d_{1 i}} \exp \{2 \varepsilon(s-t)\} x^{T}(s) R_{1} x(s) d s \\
& +\int_{t-d_{1 i}}^{t} \exp \{2 \varepsilon(s-t)\} x^{T}(s) R_{2} x(s) d s \\
& +\int_{t-d_{m i}}^{t-d_{1 i}} \exp \{2 \varepsilon(s-t)\} x^{T}(s) R_{3} x(s) d s \\
& +\int_{t-d_{2 i}}^{t-d_{m i}} \exp \{2 \varepsilon(s-t)\} x^{T}(s) R_{4} x(s) d s, \\
& V_{4}\left(x_{t}, i\right)=\int_{t-d_{i}(t)}^{t-d_{1 i}} \exp \{2 \varepsilon(s-t)\} \dot{x}^{T}(s) T_{1} \dot{x}(s) d s \\
& +\int_{t-d_{1 i}}^{t} \exp \{2 \varepsilon(s-t)\} \dot{x}^{T}(s) T_{2} \dot{x}(s) d s \\
& +\int_{t-d_{m i}}^{t-d_{1 i}} \exp \{2 \varepsilon(s-t)\} \dot{x}^{T}(s) T_{3} \dot{x}(s) d s \\
& +\int_{t-d_{2 i}}^{t-d_{m i}} \exp \{2 \varepsilon(s-t)\} \dot{x}^{T}(s) T_{4} \dot{x}(s) d s
\end{aligned}
$$

and $V_{5}\left(x_{t}, i\right), V_{6}\left(x_{t}, i\right), V_{7}\left(x_{t}, i\right)$ are the same as (43), (44), and (45).

Then, we follow a similar line as in proof of Theorem 10 and obtain the result.

3.2. Extension to the Uncertain Case. In this subsection, the uncertain neutral Markovian jump systems described by (6) with partially unknown transition rates are considered. The delay-range-dependent and rate-dependent exponential stability conditions are presented in the following theorems and corollaries.

Theorem 18. For given scalars $\pi_{i}^{m}, \pi_{i}^{M}, \alpha, \beta, \gamma, \varepsilon, \overline{\tau_{i}}, v_{i}, d_{1 i}$, $d_{2 i}, \mu_{i}$ and constant scalar $d_{m i}$ satisfying $d_{1 i}<d_{m i}<d_{2 i}$, the systems described by (6) with partially known transition rates are exponentially stable with decay rate $\varepsilon$ and $\kappa=\sqrt{\overline{\bar{\lambda}} / \underline{\lambda}}$ if $\left\|C_{i}\right\|+\gamma<1$ and there exist scalars $\delta_{1}>0, \delta_{2}>0$, symmetric positive matrices $P_{i}>0, Q_{1 i}>0, Q_{2 i}>0, R_{1 i}>0, T_{1 i}>0$, $(i \in S), Q_{j}>0,(j=3,4), R_{k}>0, T_{l}>0,(k, l=2,3,4)$, $U_{m}>0, V_{n}>0, W_{s}>0,(m, n, s=1,2,3,4)$ and matrices $M_{1}$, $M_{2}, N_{1}, N_{2}, N_{3}, N_{4}$ for any scalars $\varepsilon_{1}, \varepsilon_{2}, \varepsilon_{3}$, any symmetric matrices $X_{i}, Y_{1 i}, Y_{2 i}, Z_{1 i}, Z_{2 i},(i \in S)$, and any matrices $J_{k}$, $(k=1,2, \ldots, 24)$ with appropriate dimensions, such that (26)-

(32) and the following inequalities hold:

$$
\begin{aligned}
& \text { (i) }\left[\begin{array}{cc}
U_{3} & N_{1} \\
N_{1}^{T} & U_{3}
\end{array}\right]>0, \quad\left[\begin{array}{cc}
V_{3} & N_{2} \\
N_{2}^{T} & V_{3}
\end{array}\right]>0, \\
& \text { (ii) }\left[\begin{array}{cc}
\delta_{1} \Omega_{i 0}^{\varepsilon}+\delta_{1} \Omega_{i 1}^{\varepsilon}+\delta_{1}^{2} \mathbb{M}^{T} \mathbb{M} & \mathbb{N}^{T} \\
* & -I
\end{array}\right]<0, \\
& \text { (iii) }\left[\begin{array}{ll}
U_{4} & N_{3} \\
N_{3}^{T} & U_{4}
\end{array}\right]>0, \quad\left[\begin{array}{cc}
V_{4} & N_{4} \\
N_{4}^{T} & V_{4}
\end{array}\right]>0, \\
& \text { (iv) }\left[\begin{array}{cc}
\delta_{2} \Omega_{i 0}^{\varepsilon}+\delta_{2} \Omega_{i 2}^{\varepsilon}+\delta_{2}^{2} \mathbb{M}^{T} \mathbb{M} & \mathbb{N}^{T} \\
* & -I
\end{array}\right]<0,
\end{aligned}
$$

where

$$
\begin{gathered}
\mathbb{M}=e_{1} N_{A i}^{T}+e_{3} N_{B i}^{T}+e_{22} N_{D i}^{T}+e_{23} N_{E i}^{T}+e_{24} N_{F i}^{T}, \\
\mathbb{N}=L_{i}^{T} P_{i}^{T} e_{1}+\sum_{m=1}^{24} L_{i}^{T} J_{m}^{T} e_{m}
\end{gathered}
$$

and other notations are the same as Theorem 10.

Proof. Defining $\mathbb{E}=\operatorname{col}\left\{e_{m},(m=1,2, \ldots, 24)\right\}$ and $\mathbb{\rrbracket}=$ $\operatorname{col}\left\{P_{i}+J_{1}, J_{m},(m=2, \ldots, 24)\right\}$, we replace $A_{i}, B_{i}, D_{i}, E_{i}$, and $F_{i}$ with $A_{i}+\Delta A_{i}(t), B_{i}+\Delta B_{i}(t), D_{i}+\Delta D_{i}(t), E_{i}+\Delta E_{i}(t)$, and $F_{i}+\Delta F_{i}(t)$ on the basis of Theorem 10. That is,

$$
\text { (i) }\left[\begin{array}{ll}
U_{3} & N_{1} \\
N_{1}^{T} & U_{3}
\end{array}\right]>0, \quad\left[\begin{array}{cc}
V_{3} & N_{2} \\
N_{2}^{T} & V_{3}
\end{array}\right]>0 \text {, }
$$

(ii) $\Omega_{i 0}^{\varepsilon}+\Omega_{i 1}^{\varepsilon}+\mathscr{L}\left[e_{1} \Delta A_{i}^{T}(t) \rrbracket^{T} \mathbb{E}+e_{3} \Delta B_{i}^{T}(t) \rrbracket^{T} \mathbb{E}\right.$

$$
\begin{aligned}
& +e_{22} \Delta D_{i}^{T}(t) \rrbracket^{T} \mathbb{E}+e_{23} \Delta E_{i}^{T}(t) \rrbracket^{T} \mathbb{E} \\
& \left.+e_{24} \Delta F_{i}^{T}(t) \rrbracket^{T} \mathbb{E}\right]<0,
\end{aligned}
$$

$$
\text { (iii) }\left[\begin{array}{cc}
U_{4} & N_{3} \\
N_{3}^{T} & U_{4}
\end{array}\right]>0, \quad\left[\begin{array}{cc}
V_{4} & N_{4} \\
N_{4}^{T} & V_{4}
\end{array}\right]>0 \text {, }
$$

(iv) $\Omega_{i 0}^{\varepsilon}+\Omega_{i 2}^{\varepsilon}+\mathscr{L}\left[e_{1} \Delta A_{i}^{T}(t) \rrbracket^{T} \mathbb{E}+e_{3} \Delta B_{i}^{T}(t) \rrbracket^{T} \mathbb{E}\right.$

$$
\begin{aligned}
& +e_{22} \Delta D_{i}^{T}(t) \rrbracket^{T} \mathbb{E}+e_{23} \Delta E_{i}^{T}(t) \rrbracket^{T} \mathbb{E} \\
& \left.+e_{24} \Delta F_{i}^{T}(t) \mathbb{J}^{T} \mathbb{E}\right]<0 .
\end{aligned}
$$


Considering (ii) of (99) and combining the uncertainties condition (11), we have

$$
\left.\begin{array}{rl}
\Omega_{i 0}^{\varepsilon}+ & \Omega_{i 1}^{\varepsilon}+\mathscr{L} \\
& \left.\times\left\{\left[\begin{array}{lllll}
e_{1} & e_{3} & e_{22} & e_{23} & e_{24}
\end{array}\right]\left[\begin{array}{c}
N_{A i}^{T} \\
N_{B i}^{T} \\
N_{D i}^{T} \\
N_{E i}^{T} \\
N_{F i}^{T}
\end{array}\right] H_{i}^{T}(t) L_{i}^{T}\right]^{T} \mathbb{E}\right\}<0 .
\end{array}\right]
$$

By the definition of $\mathscr{L}$, we obtain

$$
\Omega_{i 0}^{\varepsilon}+\Omega_{i 1}^{\varepsilon}+\mathbb{M} H_{i}^{T}(t) \mathbb{N}+\mathbb{N}^{T} H_{i}(t) \mathbb{M}^{T}<0 .
$$

According to (12), by Lemmas 9 and 6, with (102) we obtain (ii) of (96). Following the same procedure, (iv) of (100) is considered and (iv) of (97) can be obtained. Finally, following the proof of Theorem 10, the systems described by (6) are exponentially stable with a decay rate $\varepsilon$. This completes the proof.

Considering the uncertain case, the following corollaries are given, for $\varepsilon=0$ and entirely unknown transition rates, respectively.

Corollary 19. For given scalars $\pi_{i}^{m}, \pi_{i}^{M}, \alpha, \beta, \gamma, \overline{\tau_{i}}, v_{i}, d_{1 i}$, $d_{2 i}, \mu_{i}$ and constant scalar $d_{m i}$ satisfying $d_{1 i}<d_{m i}<d_{2 i}$, the systems described by (6) with partially known transition rates are asymptotically stable if $\left\|C_{i}\right\|+\gamma<1$ and there exist scalars $\delta_{1}>0, \delta_{2}>0$, symmetric positive matrices $P_{i}>0$, $Q_{1 i}>0, Q_{2 i}>0, R_{1 i}>0, T_{1 i}>0,(i \in S), Q_{j}>0,(j=3,4)$, $R_{k}>0, T_{l}>0,(k, l=2,3,4), U_{m}>0, V_{n}>0$, and $W_{s}>0$, $(m, n, s=1,2,3,4)$ and matrices $M_{1}, M_{2}, N_{1}, N_{2}, N_{3}, N_{4}$ for any scalars $\varepsilon_{1}, \varepsilon_{2}, \varepsilon_{3}$, any symmetric matrices $X_{i}, Y_{1 i}, Y_{2 i}$, $Z_{1 i}, Z_{2 i},(i \in S)$ and any matrices $J_{k},(k=1,2, \ldots, 24)$ with appropriate dimensions, such that (26)-(32), and the following inequalities hold:

$$
\begin{aligned}
& \text { (i) }\left[\begin{array}{cc}
U_{3} & N_{1} \\
N_{1}^{T} & U_{3}
\end{array}\right]>0, \quad\left[\begin{array}{cc}
V_{3} & N_{2} \\
N_{2}^{T} & V_{3}
\end{array}\right]>0, \\
& \text { (ii) }\left[\begin{array}{cc}
\delta_{1} \Omega_{i 0}+\delta_{1} \Omega_{i 1}+\delta_{1}^{2} \mathbb{M}^{T} \mathbb{M} & \mathbb{N}^{T} \\
* & -I
\end{array}\right]<0, \\
& \text { (iii) }\left[\begin{array}{cc}
U_{4} & N_{3} \\
N_{3}^{T} & U_{4}
\end{array}\right]>0, \quad\left[\begin{array}{cc}
V_{4} & N_{4} \\
N_{4}^{T} & V_{4}
\end{array}\right]>0, \\
& \text { (iv) }\left[\begin{array}{cc}
\delta_{2} \Omega_{i 0}+\delta_{2} \Omega_{i 2}+\delta_{2}^{2} \mathbb{M}^{T} \mathbb{M} & \mathbb{N}^{T} \\
* & -I
\end{array}\right]<0,
\end{aligned}
$$

where

$$
\begin{gathered}
\mathbb{M}=e_{1} N_{A i}^{T}+e_{3} N_{B i}^{T}+e_{22} N_{D i}^{T}+e_{23} N_{E i}^{T}+e_{24} N_{F i}^{T}, \\
\mathbb{N}=L_{i}^{T} P_{i}^{T} e_{1}+\sum_{m=1}^{24} L_{i}^{T} J_{m}^{T} e_{m}
\end{gathered}
$$

and other notations are the same as Corollary 16.
Corollary 20. For given scalars $\pi_{i}^{m}, \pi_{i}^{M}, \alpha, \beta, \gamma, \varepsilon, \overline{\tau_{i}}, v_{i}, d_{1 i}$, $d_{2 i}, \mu_{i}$ and constant scalar $d_{m i}$ satisfying $d_{1 i}<d_{m i}<d_{2 i}$, the systems described by (6) with completely unknown transition rates are exponentially stable with decay rate $\varepsilon$ and $\kappa=\sqrt{\bar{\lambda}} / \underline{\lambda}$ if $\left\|C_{i}\right\|+\gamma<1$ and there exist scalars $\delta_{1}>0, \delta_{2}>0$, symmetric positive matrices $P>0, Q_{j}>0, R_{k}>0, T_{l}>0, U_{m}>0, V_{n}>$ $0, W_{s}>0,(j, k, l, m, n, s=1,2,3,4)$ and matrices $M_{1}, M_{2}, N_{1}$, $N_{2}, N_{3}, N_{4}$ for any scalars $\varepsilon_{1}, \varepsilon_{2}, \varepsilon_{3}$, any symmetric matrices $Y_{1}, Y_{2}, Z_{1}, Z_{2}$, and any matrices $J_{k},(k=1,2, \ldots, 24)$ with appropriate dimensions, such that (86), (87), and the following inequalities hold:

$$
\begin{aligned}
& \text { (i) }\left[\begin{array}{ll}
U_{3} & N_{1} \\
N_{1}^{T} & U_{3}
\end{array}\right]>0, \quad\left[\begin{array}{cc}
V_{3} & N_{2} \\
N_{2}^{T} & V_{3}
\end{array}\right]>0 \text {, } \\
& \text { (ii) }\left[\begin{array}{cc}
\delta_{1} \widetilde{\Omega}_{i 0}^{\varepsilon}+\delta_{1} \widetilde{\Omega}_{i 1}^{\varepsilon}+\delta_{1}^{2} \mathbb{M}^{T} \mathbb{M} & \mathbb{N}^{T} \\
* & -I
\end{array}\right]<0 \text {, } \\
& \text { (iii) }\left[\begin{array}{cc}
U_{4} & N_{3} \\
N_{3}^{T} & U_{4}
\end{array}\right]>0, \quad\left[\begin{array}{cc}
V_{4} & N_{4} \\
N_{4}^{T} & V_{4}
\end{array}\right]>0 \text {, } \\
& \text { (iv) }\left[\begin{array}{cc}
\delta_{2} \widetilde{\Omega}_{i 0}^{\varepsilon}+\delta_{2} \widetilde{\Omega}_{i 2}^{\varepsilon}+\delta_{2}^{2} \mathbb{M}^{T} \mathbb{M} & \mathbb{N}^{T} \\
* & -I
\end{array}\right]<0,
\end{aligned}
$$

where

$$
\begin{gathered}
\mathbb{M}=e_{1} N_{A i}^{T}+e_{3} N_{B i}^{T}+e_{22} N_{D i}^{T}+e_{23} N_{E i}^{T}+e_{24} N_{F i}^{T}, \\
\mathbb{N}=L_{i}^{T} P_{i}^{T} e_{1}+\sum_{m=1}^{24} L_{i}^{T} J_{m}^{T} e_{m}
\end{gathered}
$$

and other notations are the same as Corollary 17.

\section{Numerical Examples}

In this section, numerical examples are given to demonstrate that the proposed theoretical results in this paper are effectiveness. In particular, when $i \in S=\{1\}, d_{i}(t), d_{1 i}, d_{m i}$, and $d_{2 i}$ are expressed into $d(t), d_{1}, d_{m}$, and $d_{2}$.

Example 1. We consider an interval mode-dependent timevarying delay system in the form of (6) with three modes and nonlinear perturbations:

$$
\begin{gathered}
\left\|f_{1}(x(t), t)\right\| \leq 0.1\|x(t)\|, \\
\left\|f_{2}(x(t-d(t)), t)\right\| \leq 0.2\|x(t-d(t))\|, \\
\left\|f_{3}(\dot{x}(t-\tau(t)), t)\right\| \leq 0.1\|\dot{x}(t-\tau(t))\| ;
\end{gathered}
$$

that is, $\alpha=0.1, \beta=0.2, \gamma=0.1$. The parametric matrices of the system are given as follows:

$$
\begin{array}{rlrl}
A_{1} & =\left[\begin{array}{cc}
-0.75 & -0.75 \\
1.50 & -1.50
\end{array}\right], & A_{2}=\left[\begin{array}{cc}
-0.15 & -0.09 \\
1.50 & -0.10
\end{array}\right], \\
A_{3}=\left[\begin{array}{cc}
-0.30 & -0.15 \\
0.50 & -0.50
\end{array}\right], & B_{1}=\left[\begin{array}{cc}
0.11 & 0.23 \\
-0.52 & -0.37
\end{array}\right], \\
B_{2}=\left[\begin{array}{cc}
-0.59 & 0.02 \\
-0.06 & -0.61
\end{array}\right], & B_{3}=\left[\begin{array}{cc}
0.51 & 0.24 \\
0.02 & -0.44
\end{array}\right],
\end{array}
$$



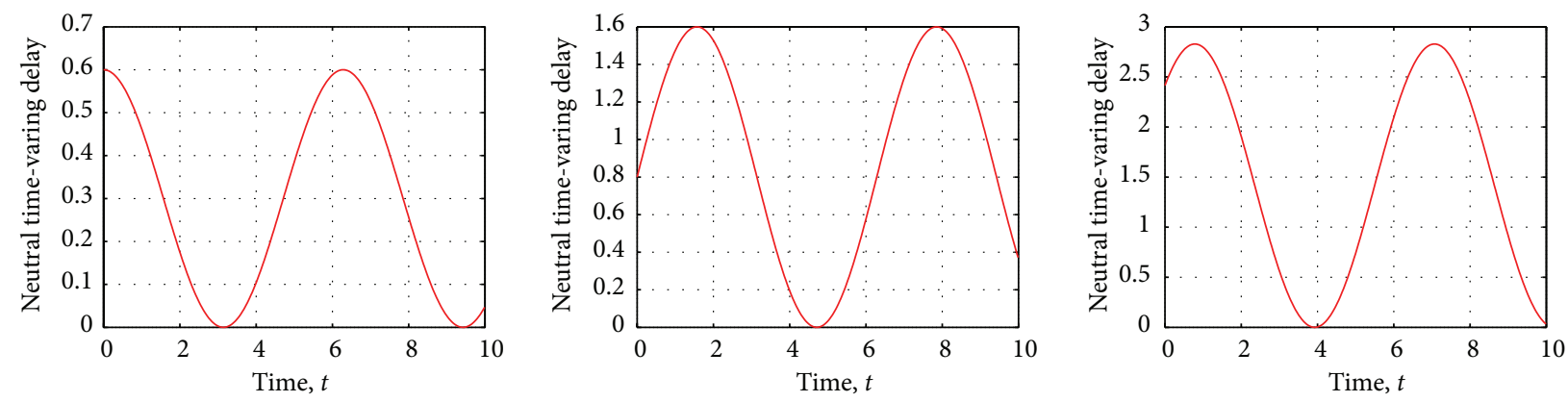

Figure 1: Neutral time-varying delay $\tau_{i}(t)$ at Mode 1, Mode 2, and Mode 3.

$$
\begin{gathered}
C_{1}=\left[\begin{array}{cc}
0.30 & 0 \\
0 & 0.20
\end{array}\right], \quad C_{2}=\left[\begin{array}{cc}
0.10 & 0 \\
0 & 0.20
\end{array}\right] \\
C_{3}=\left[\begin{array}{cc}
0.20 & 0 \\
0 & 0.40
\end{array}\right], \quad D_{1}=\left[\begin{array}{cc}
-0.70 & -0.34 \\
0.50 & -0.65
\end{array}\right], \\
D_{2}=\left[\begin{array}{cc}
-0.67 & -0.50 \\
0.39 & 0.23
\end{array}\right], \quad D_{3}=\left[\begin{array}{cc}
-0.23 & 0.16 \\
0.02 & -0.57
\end{array}\right], \\
E_{1}=\left[\begin{array}{cc}
-0.30 & -0.44 \\
0.50 & -0.65
\end{array}\right], \quad E_{2}=\left[\begin{array}{cc}
-0.40 & -0.35 \\
0.39 & 0.28
\end{array}\right], \\
E_{3}=\left[\begin{array}{cc}
-0.24 & 0.19 \\
0.12 & -0.38
\end{array}\right], \quad F_{1}=\left[\begin{array}{cc}
-0.50 & -0.36 \\
0.26 & -0.75
\end{array}\right], \\
F_{2}=\left[\begin{array}{cc}
-0.48 & -0.33 \\
0.27 & 0.22
\end{array}\right], \quad F_{3}=\left[\begin{array}{cc}
-0.45 & 0.23 \\
0.12 & -0.35
\end{array}\right], \\
L_{1}=L_{2}=L_{3}=[0.1 \\
N_{A 1}=N_{A 2}=N_{A 3}=\left[\begin{array}{l}
0.1 \\
0.1
\end{array}\right], \\
N_{E 1}=N_{E 2}=N_{E 3}=\left[\begin{array}{l}
0.2 \\
0.3
\end{array}\right], \\
N_{B 1}=N_{B 2}=N_{B 3}=\left[\begin{array}{l}
0.1 \\
0.2
\end{array}\right],
\end{gathered}
$$

The partially known transition rates matrix is described by

$$
\left[\begin{array}{ccc}
-0.8 & ? & ? \\
? & -0.9 & ? \\
0.7 & 0.4 & -1.1
\end{array}\right]
$$

where $\pi_{i}^{m}=-1.1, \pi_{i}^{M}=-0.8$.

The interval mode-dependent time-varying neutral delays are assumed to be

$$
\begin{gathered}
\tau_{1}(t)=0.3(1+\cos t), \quad \tau_{2}(t)=0.8(1+\sin t), \\
\tau_{3}(t)=\sqrt{2}+\sin t+\cos t .
\end{gathered}
$$

The interval mode-dependent time-varying retarded delays are assumed to be

$$
\begin{gathered}
d_{1}(t)=0.4\left(1+\sin ^{2}(3 t)\right), \quad d_{2}(t)=0.5\left(1+\cos ^{2}(4 t)\right), \\
d_{3}(t)=0.6 \sin ^{4} t .
\end{gathered}
$$

They are governed by the Markov process $\left\{r_{t}, t \geq 0\right\}$ and shown in Figures 1 and 2. It can be readily obtain that

$$
\begin{gathered}
\overline{\tau_{1}}=0.6, \quad \overline{\tau_{2}}=1.6, \quad \overline{\tau_{3}}=2 \sqrt{2} ; \\
v_{1}=0.3, \quad v_{2}=0.8, \quad v_{3}=\sqrt{2} ; \\
d_{11}=0.4, \quad d_{21}=0.8 ; \quad d_{12}=0.5, \quad d_{22}=1 ; \\
d_{13}=0, \quad d_{23}=0.6 ; \\
\mu_{1}=1.2, \quad \mu_{2}=2, \quad \mu_{3}=\frac{9 \sqrt{3}}{20} .
\end{gathered}
$$

Providing that decay rate $\varepsilon=0.5$, we choose qualified values of $d_{m 1}, d_{m 2}, d_{m 3}$, for example, $d_{m 1}=0.5, d_{m 2}=$ $0.7, d_{m 3}=0.3$, and then we solve (26)-(32), (96), and (97) in Theorem 18. Thus, it is facile to establish the exponential stability of this uncertain neutral system described by (6), which shows that the approach presented in this paper is effectiveness.

Example 2. Consider the nominal system (13) with four operation modes $S=\{1,2,3,4\}, \alpha=\beta=\gamma=0$, and the following parameters:

$$
\begin{aligned}
A_{1}=\left[\begin{array}{cc}
-1.15 & -0.75 \\
1.50 & -1.50
\end{array}\right], & B_{1}=\left[\begin{array}{cc}
-1.20 & 0.12 \\
0.24 & -0.25
\end{array}\right], \\
C_{1}=\left[\begin{array}{cc}
-0.15 & -0.06 \\
0.50 & -0.50
\end{array}\right], & A_{2}=\left[\begin{array}{cc}
-2.15 & -0.49 \\
1.50 & -2.10
\end{array}\right], \\
B_{2}=\left[\begin{array}{cc}
-1.45 & -0.96 \\
0.47 & -1.57
\end{array}\right], & C_{2}=\left[\begin{array}{cc}
0.25 & -0.35 \\
0.05 & -0.65
\end{array}\right], \\
A_{3}=\left[\begin{array}{cc}
-1.30 & -0.15 \\
1.50 & -1.80
\end{array}\right], & B_{3}=\left[\begin{array}{cc}
0.58 & -0.68 \\
-0.13 & 0.96
\end{array}\right],
\end{aligned}
$$



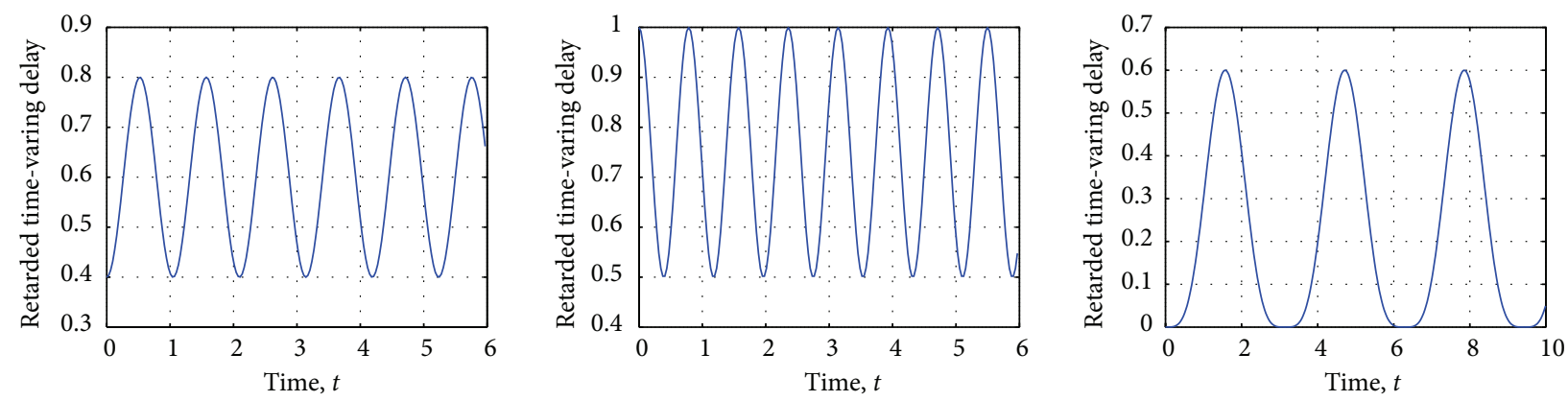

Figure 2: Retarded time-varying delay $d_{i}(t)$ at Mode 1, Mode 2, and Mode 3.

TABLE 1: Maximum upper bound of $d_{2}$ with $\Pi_{1}$ and different parameter $\mu$.

\begin{tabular}{lccccccccc}
\hline Methods & $\mu$ & 0.18 & 0.19 & 0.20 & 0.21 & 0.22 & 0.23 & 0.24 & 0.25 \\
\hline Xiong et al. [40] & $d_{2}$ & 0.4489 & 0.3317 & 0.2242 & 0.1814 & 0.1486 & 0.1220 & 0.0983 & 0.0781 \\
Corollary 16 & $d_{2}$ & 0.4771 & 0.3664 & 0.2583 & 0.2165 & 0.1897 & 0.1473 & 0.1144 & 0.0956 \\
\hline
\end{tabular}

$$
\begin{array}{rlr}
C_{3} & =\left[\begin{array}{cc}
0.33 & 0.07 \\
0.19 & -0.36
\end{array}\right], & A_{4}=\left[\begin{array}{cc}
-1.90 & -0.34 \\
1.50 & -1.65
\end{array}\right], \\
B_{4}=\left[\begin{array}{cc}
-0.67 & -1.50 \\
1.39 & 1.23
\end{array}\right], & C_{4}=\left[\begin{array}{cc}
-0.23 & 0.16 \\
0.02 & -0.57
\end{array}\right] .
\end{array}
$$

The partially known transition rate matrix is considered as the following two cases:

$$
\begin{aligned}
& \Pi_{1}=\left[\begin{array}{cccc}
-1.3 & 0.2 & ? & ? \\
? & ? & 0.3 & 0.3 \\
0.6 & ? & -1.5 & ? \\
0.4 & ? & ? & ?
\end{array}\right], \\
& \Pi_{2}=\left[\begin{array}{cccc}
-1.3 & 0.2 & 0.4 & 0.7 \\
? & ? & 0.3 & 0.3 \\
0.6 & ? & -1.5 & ? \\
0.4 & ? & ? & ?
\end{array}\right],
\end{aligned}
$$

where $\pi_{i}^{m}=-1.8$ and $\pi_{i}^{M}=-1.3$.

Providing that $\varepsilon=0$ and $\tau_{i}(t)=d_{i}(t)=d(t), i \in S=$ $\{1,2,3,4\}$, we have $d_{1}=0, \bar{\tau}=d_{2}, v=\mu$. Set $d_{m}=d_{2} / 2$ and employ Corollary 16; the maximum upper bound of the time delay $d_{2}$ which satisfies LMIs (26)-(32), and (83), can be calculated by solving a quasi-convex optimization problem. This neutral Markovian jump system with partially unknown transition rates was also considered in [40]. The results on the maximum upper bound of $d_{2}$ are compared in Tables 1 and 2 .

From Tables 1 and 2, we consider the previous system with $\Pi_{1}, \Pi_{2}$ and obtain the maximum upper bound of delay $d_{2}=0.4771, d_{2}=12.4394$, respectively, in this paper by setting $\mu=0.18$, while the maximum upper bound of delay $d_{2}=0.4489, d_{2}=12.0750$, respectively, for [40]. The results are also given by setting $\mu=0.19, \mu=0.20, \mu=0.21$, $\mu=0.22, \mu=0.23, \mu=0.24$, and $\mu=0.25$, and it is found that the maximum upper bound of delay in this paper is larger than that of [40]. So it can be seen that our proposed method is less conservative than the result in [40]. Besides, according to the data in Tables 1 and 2, we know that the maximum upper bound of delay to guarantee stability is dependent on transition rates knowledge.

Example 3. To show the reduced conservativeness of the exponential stability condition in Theorem 10, consider the time-delay system in the form of (13) with $C_{i}=0$ and $\alpha=$ $\beta=\gamma=0, d_{i}(t)=d$ and

$$
A_{i}=\left[\begin{array}{cc}
-3 & -2 \\
1 & 0
\end{array}\right], \quad B_{i}=\left[\begin{array}{cc}
-0.5 & 0.1 \\
0.3 & 0
\end{array}\right], \quad i \in S=\{1\}
$$

For given $d$, the maximum exponential decay rate $\varepsilon$, which satisfies the LMIs (32), (33), and (34) in Theorem 10, can be calculated by solving a quasi-convex optimization problem. The results are presented in Table 3.

From Table 3, we know that the maximum exponential decay rate $\varepsilon=0.9569$ in this paper by setting $d=0.8$, while the maximum exponential decay rate $\varepsilon=0.7344$ for [41], $\varepsilon=$ 0.9367 for [42], and $\varepsilon=0.9366$ for [43]. The results are also given by setting $d=1.0, d=1.2, d=1.4, d=1.6, d=1.8$, and $d=2.0$, and it is found that the maximum exponential decay rate in this paper is larger than those in [41-43]. So it can be demonstrated that Theorem 10 in this paper yields less conservative results than [41-43].

Consider the previous system again, but with parametric matrices and parameter uncertainties as follows:

$$
A_{i}=\left[\begin{array}{cc}
-4 & 1 \\
0 & -4
\end{array}\right], \quad B_{i}=\left[\begin{array}{cc}
0.1 & 0 \\
4 & 0.1
\end{array}\right], \quad i \in S=\{1\}
$$

and the uncertain matrices $\Delta A_{i}(t)$ and $\Delta B_{i}(t)$ satisfy

$$
\begin{array}{r}
L_{i}=0.2 I, \quad N_{A i}=N_{B i}=I, \quad\left\|H_{i}(t)\right\|<1, \\
i \in S=\{1\} .
\end{array}
$$


TABLE 2: Maximum upper bound of $d_{2}$ with $\Pi_{2}$ and different parameter $\mu$.

\begin{tabular}{lccccccccc}
\hline Methods & $\mu$ & 0.18 & 0.19 & 0.20 & 0.21 & 0.22 & 0.23 & 0.24 & 0.25 \\
\hline Xiong et al. [40] & $d_{2}$ & 12.0750 & 0.9924 & 0.5147 & 0.3749 & 0.2742 & 0.2139 & 0.1737 & 0.1433 \\
Corollary 16 & $d_{2}$ & 12.4394 & 1.2587 & 0.6263 & 0.4037 & 0.3003 & 0.2485 & 0.2018 & 0.1796 \\
\hline
\end{tabular}

TABLE 3: Maximum upper bound of $\varepsilon$ with different parameter $d$.

\begin{tabular}{lcccccccc}
\hline Methods & $d$ & 0.8 & 1 & 1.2 & 1.4 & 1.6 & 1.8 & 2.0 \\
\hline Mondié and Kharitonov [41] & $\varepsilon$ & 0.7344 & 0.6715 & 0.6145 & 0.5642 & 0.5202 & 0.4818 & 0.4481 \\
Liu [42] & $\varepsilon$ & 0.9367 & 0.5903 & 0.3400 & 0.1813 & 0.0752 & 0.0014 & 0 \\
Xu et al. [43] & $\varepsilon$ & 0.9366 & 0.9192 & 0.8991 & 0.8115 & 0.6990 & 0.6148 & 0.5494 \\
Theorem 10 & $\varepsilon$ & 0.9569 & 0.9407 & 0.9218 & 0.9024 & 0.8154 & 0.7394 & 0.6933 \\
\hline
\end{tabular}

TABLE 4: Maximum upper bound of $\varepsilon$ with different parameter $d$.

\begin{tabular}{lcccccccc}
\hline Methods & $d$ & 0.3 & 0.5 & 0.7 & 0.9 & 1.1 & 1.3 & 1.5 \\
\hline Mondié and Kharitonov [41] & $\varepsilon$ & 0.6255 & 0.4760 & 0.3825 & 0.3191 & 0.2735 & 0.2392 & 0.2125 \\
Xu et al. [43] & $\varepsilon$ & 1.0108 & 0.8366 & 0.7103 & 0.6156 & 0.5425 & 0.4845 & 0.4375 \\
Theorem 18 & $\varepsilon$ & 1.0419 & 0.9207 & 0.8035 & 0.7048 & 0.6234 & 0.5876 & 0.5043 \\
\hline
\end{tabular}

For given $d$, the maximum exponential decay rate $\varepsilon$, which satisfies the LMIs in (32), (96), and (97) in Theorem 18, can be calculated by solving a quasi-convex optimization problem. The results are presented in Table 4 and it also can be seen that the delay-range-dependent and rate-dependent exponential stability conditions in this paper are less conservative than previous results in $[41,43]$.

Example 4. Consider the system (6) with $\tau_{i}(t)=d_{i}(t)=d(t)$ and the parameters are listed in the following:

$$
\begin{gathered}
A_{i}=\left[\begin{array}{cc}
-2 & 0 \\
0 & -0.9
\end{array}\right], \quad B_{i}=\left[\begin{array}{cc}
-1 & 0 \\
-1 & -1
\end{array}\right], \quad C_{i}=\left[\begin{array}{cc}
0.1 & 0 \\
0 & 0.1
\end{array}\right], \\
D_{i}=E_{i}=F_{i}=I, \quad i \in S=\{1\}, \\
\alpha=0.05, \quad \beta=0.1, \quad \gamma=0, \\
d_{1}=0, \quad d_{2}=\bar{\tau}, \quad \mu=\nu .
\end{gathered}
$$

For given $\varepsilon$ and $\mu$, choose $d_{m}=d_{2} / 2$ and utilize Theorem 10, the maximum upper bound of $d_{2}$, which satisfies the LMIs in (32), (33), and (34), can be obtained by solving a quasi-convex optimization problem. The results are presented in Tables 5, 6 , and 7.

From Tables 5, 6, and 7, we consider $\mu=0, \mu=0.5$, and $\mu=0.9$ and obtain the maximum upper bound of delay $d_{2}=1.5167, d_{2}=1.0643$, and $d_{2}=0.7136$, respectively, in this paper by setting $\varepsilon=0.1$, while the maximum upper bound of delay $d_{2}=1.2999, d_{2}=0.9442$, and $d_{2}=0.5471$, respectively, for [44], the maximum upper bound of delay $d_{2}=1.4008, d_{2}=1.0120$, and $d_{2}=0.6438$, respectively, for [13]. The results are also given by setting $\varepsilon=0.3, \varepsilon=0.5$, $\varepsilon=0.7$, and $\varepsilon=0.9$, and it is found that the maximum upper bound of delay in this paper is larger than those in $[13,44]$.
According to the comparative result, it can be seen that our proposed method is less conservative than those in $[13,44]$.

Example 5. Partial element equivalent circuit (PEEC) model can be represented as a stochastic jump system as in (6) with the abrupt variation in structures and parameters [47]. Consider the practical PEEC system described by (6) with $\alpha=\beta=\gamma=0$ and completely unknown transition rates

$$
\Pi=\left[\begin{array}{ll}
? & ? \\
? & ?
\end{array}\right], \quad i \in S=\{1,2\}
$$

where $\pi_{i}^{m}=-1.0$ and $\pi_{i}^{M}=-0.6$.

The parametric matrices of the system are given as follows:

$$
\begin{array}{cc}
A_{1}=\left[\begin{array}{cc}
-4 & 0 \\
0 & -3
\end{array}\right], & A_{2}=\left[\begin{array}{cc}
-2 & 0 \\
0 & -3
\end{array}\right], \\
B_{1}=\left[\begin{array}{cc}
-1 & 0 \\
-1 & -1
\end{array}\right], & B_{2}=\left[\begin{array}{cc}
-1 & 0 \\
-0.9 & -1.2
\end{array}\right], \\
C_{1}=0.5 I, & C_{2}=0.3 I, \\
L_{1}=\left[\begin{array}{l}
0.2 \\
0.2
\end{array}\right], & L_{2}=\left[\begin{array}{c}
0 \\
-0.3
\end{array}\right], \\
N_{A 1}=\left[\begin{array}{ll}
0.2 & 0
\end{array}\right], & N_{A 2}=\left[\begin{array}{ll}
0 & 0.2
\end{array}\right], \\
N_{B 1}=\left[\begin{array}{ll}
-0.3 & 0.3
\end{array}\right], & N_{B 2}=\left[\begin{array}{ll}
0.2 & 0.2
\end{array}\right] .
\end{array}
$$

Given the decay rate $\varepsilon=0.4$, the bound of mode-dependent time-varying neutral delay $0 \leq \tau_{1}(t) \leq 0.5,0 \leq \tau_{2}(t) \leq 0.6$, and the bound of mode-dependent time-varying retarded delay $0.1 \leq d_{1}(t) \leq 0.2,0.3 \leq d_{2}(t) \leq 0.4$. Without loss of generality, we choose $d_{m 1}=0.12$ and $d_{m 2}=0.33$. Since the information on the delay derivative is not available, by setting $Q_{1}=Q_{2}=0, R_{1}=0, T_{1}=0$ in the Lyapunov 
TABLe 5: Maximum upper bound of $d_{2}$ with different $\varepsilon$ and parameter $\mu=0$.

\begin{tabular}{lccccrr}
\hline Methods & $\varepsilon$ & 0.1 & 0.3 & 0.5 & 0.7 & 0.9 \\
\hline Chen et al. [44] & $d_{2}$ & 1.2999 & 0.8781 & 0.6917 & 0.5792 & 0.5015 \\
Qiu and Cui [13] & $d_{2}$ & 1.4008 & 1.0199 & 0.8457 & 0.7395 & 0.6667 \\
Theorem 10 & $d_{2}$ & 1.5167 & 1.1365 & 1.0674 & 0.9677 & 0.7558 \\
\hline
\end{tabular}

TABLE 6: Maximum upper bound of $d_{2}$ with different $\varepsilon$ and parameter $\mu=0.5$.

\begin{tabular}{lccccrr}
\hline Methods & $\varepsilon$ & 0.1 & 0.3 & 0.5 & 0.7 & 0.9 \\
\hline Chen et al. [44] & $d_{2}$ & 0.9442 & 0.7275 & 0.6096 & 0.5321 & 0.4761 \\
Qiu and Cui [13] & $d_{2}$ & 1.0120 & 0.8324 & 0.7311 & 0.6941 & 0.6063 \\
Theorem 10 & $d_{2}$ & 1.0643 & 0.9156 & 0.7985 & 0.7368 & 0.6849 \\
\hline
\end{tabular}

TABLE 7: Maximum upper bound of $d_{2}$ with different $\varepsilon$ and parameter $\mu=0.9$.

\begin{tabular}{lllllrr}
\hline Methods & $\varepsilon$ & 0.1 & 0.3 & 0.5 & 0.7 & 0.9 \\
\hline Chen et al. [44] & $d_{2}$ & 0.5471 & 0.5015 & 0.4650 & 0.4350 & 0.4089 \\
Qiu and Cui [13] & $d_{2}$ & 0.6438 & 0.5789 & 0.5214 & 0.4954 & 0.4231 \\
Theorem 10 & $d_{2}$ & 0.7136 & 0.6344 & 0.5987 & 0.5473 & 0.4963 \\
\hline
\end{tabular}

functional (92), we follow the same procedure as in the proof of Theorem 10 and obtain the corresponding result. Thus, the exponential stability of the practical PEEC system can be readily established, which shows the effectiveness of the approach presented in this paper.

\section{Conclusions}

The problem of exponential stability for neutral Markovian jumping systems with interval mode-dependent timevarying delays, nonlinear perturbations, and partially unknown transition rates is investigated in this paper. A novel augmented stochastic Lyapunov-Krasovskii functional is constructed, which contains some triple-integral terms and sufficiently takes advantage of the delay bound. Then, less conservative delay-range-dependent and rate-dependent exponential stability criteria are obtained by novel technique of matrix inequalities and free weighting matrices. These theoretical results are successfully verified through some numerical examples. Finally, the main contributions of this paper can be summarized as follows: (1) the constructed stochastic Lyapunov functional contains some tripleintegral terms which are very effective in the reduction of conservativeness and has not appeared in the context of neutral Markovian jump systems with partially known transition rates and nonlinear perturbations before; (2) the bound of the delay is fully utilized in this paper; that is, improved bounding technique is used to reduce the conservativeness; (3) the reciprocally convex lemma is used to derive the delay-range-dependent and ratedependent stability conditions, which can well reduce the conservativeness of the investigated systems; and (4) the proposed results are applicable to the partially known transition rates and expressed in a new representation, which are proved to be less conservative than some existing ones.

\section{Acknowledgment}

This work was supported in part by the National Key Scientific Research Project (61233003), the National Natural Science Foundation of China (60935001, 61174061, 61074033, and 60934006), the Doctoral Fund of Ministry of Education of China (20093402110019), Anhui Provincial Natural Science Foundation (11040606M143), and the Fundamental Research Funds for the Central Universities and the Program for New Century Excellent Talents in University.

\section{References}

[1] V. B. Kolmanovskii and A. Myshkis, Applied Theory of Functional Differential Equations, Kluwer Academic Publishers, Dodrecht, The Netherlands, 1992.

[2] S.-I. Niculescu, Delay Effects on Stability: A Robust Control Approach, vol. 269 of Lecture Notes in Control and Information Sciences, Springer, London, UK, 2001.

[3] P. L. Liu, "Further results on the exponential stability criteria for time delay singular systems with delay-dependence," International Journal of Innovative Computing, Information and Control, vol. 8, no. 6, pp. 4015-4024, 2012.

[4] P. L. Liu, "Improved delay-dependent robust exponential stabilization criteria for uncertain time-varying delay singular systems," International Journal of Innovative Computing, Information and Control, vol. 9, no. 1, pp. 165-178, 2013.

[5] C.-H. Lien and J.-D. Chen, "Discrete-delay-independent and discrete-delay-dependent criteria for a class of neutral systems," Journal of Dynamic Systems, Measurement and Control, Transactions of the ASME, vol. 125, no. 1, pp. 33-41, 2003.

[6] J. Tian and S. Zhong, "Improved delay-dependent stability criterion for neural networks with time-varying delay," Applied Mathematics and Computation, vol. 217, no. 24, pp. 10278-10288, 2011. 
[7] J. Tian and X. Zhou, "Improved asymptotic stability criteria for neural networks with interval time-varying delay," Expert Systems with Applications, vol. 37, no. 12, pp. 7521-7525, 2010.

[8] J. Tian and X. Xie, "New asymptotic stability criteria for neural networks with time-varying delay," Physics Letters A, vol. 374, no. 7, pp. 938-943, 2010.

[9] M. N. A. Parlakçı, "Delay-dependent robust stability criteria for uncertain neutral systems with mixed time-varying discrete and neutral delays," Asian Journal of Control, vol. 9, no. 4, pp. 411421, 2007.

[10] O. M. Kwon and J. H. Park, "Delay-range-dependent stabilization of uncertain dynamic systems with interval time-varying delays," Applied Mathematics and Computation, vol. 208, no. 1, pp. 58-68, 2009.

[11] S. Xu and J. Lam, "A survey of linear matrix inequality techniques in stability analysis of delay systems," International Journal of Systems Science, vol. 39, no. 12, pp. 1095-1113, 2008.

[12] C.-C. Shen and S.-M. Zhong, "New delay-dependent robust stability criterion for uncertain neutral systems with timevarying delay and nonlinear uncertainties," Chaos, Solitons \& Fractals, vol. 40, no. 5, pp. 2277-2285, 2009.

[13] F. Qiu and B.-T. Cui, "Improved exponential stability criteria for uncertain neutral system with nonlinear parameter perturbations," International Journal of Automation and Computing, vol. 7, no. 4, pp. 413-418, 2010.

[14] G. Zhang, C. Han, Y. Guan, and L. Wu, "Exponential stability analysis and stabilization of discrete-time nonlinear switched systems with time delays," International Journal of Innovative Computing, Information and Control, vol. 8, no. 3A, pp. 19731986, 2012.

[15] H. R. Karimi, M. Zapateiro, and N. Luo, "Stability analysis and control synthesis of neutral systems with time-varying delays and nonlinear uncertainties," Chaos, Solitons \& Fractals, vol. 42, no. 1, pp. 595-603, 2009.

[16] J. H. Park, "Novel robust stability criterion for a class of neutral systems with mixed delays and nonlinear perturbations," Applied Mathematics and Computation, vol. 161, no. 2, pp. 413421, 2005.

[17] J. Zhang, P. Shi, and J. Qiu, "Robust stability criteria for uncertain neutral system with time delay and nonlinear uncertainties," Chaos, Solitons \& Fractals, vol. 38, no. 1, pp. 160-167, 2008.

[18] Y. He, Q.-G. Wang, L. Xie, and C. Lin, "Further improvement of free-weighting matrices technique for systems with timevarying delay," IEEE Transactions on Automatic Control, vol. 52, no. 2, pp. 293-299, 2007.

[19] A. Tanikawa, "On new smoothing algorithms for discretetime linear stochastic systems with unknown disturbances," International Journal of Innovative Computing, Information and Control, vol. 4, no. 1, pp. 15-24, 2008.

[20] B. Chen, H. Li, P. Shi, C. Lin, and Q. Zhou, "Delay-dependent stability analysis and controller synthesis for Markovian jump systems with state and input delays," Information Sciences, vol. 179, no. 16, pp. 2851-2860, 2009.

[21] J. Liu, Z. Gu, and S. Hu, " $H_{\infty}$ filtering for Markovian jump systems with time-varying delays," International Journal of Innovative Computing, Information and Control, vol. 7, no. 3, pp. 1299-1310, 2011.

[22] J. Xiong and J. Lam, "Stabilization of discrete-time Markovian jump linear systems via time-delayed controllers," Automatica, vol. 42 , no. 5, pp. 747-753, 2006.
[23] J. Wu, T. Chen, and L. Wang, "Delay-dependent robust stability and $H_{\infty}$ control for jump linear systems with delays," Systems \& Control Letters, vol. 55, no. 11, pp. 939-948, 2006.

[24] P. Shi, Y. Xia, G. P. Liu, and D. Rees, "On designing of slidingmode control for stochastic jump systems," IEEE Transactions on Automatic Control, vol. 51, no. 1, pp. 97-103, 2006.

[25] S. Xu, J. Lam, and X. Mao, "Delay-dependent $H_{\infty}$ control and filtering for uncertain Markovian jump systems with timevarying delays," IEEE Transactions on Circuits and Systems I, vol. 54, no. 9, pp. 2070-2077, 2007.

[26] J. Tian, Y. Li, J. Zhao, and S. Zhong, "Delay-dependent stochastic stability criteria for Markovian jumping neural networks with mode-dependent time-varying delays and partially known transition rates," Applied Mathematics and Computation, vol. 218, no. 9, pp. 5769-5781, 2012.

[27] L. L. Xiong, X. B. Zhou, J. Qiu, and J. Lei, "Stability analysis for Markovian jump neutral systems with mixed delays and partially known transition rates," Abstract and Applied Analysis, vol. 2012, Article ID 450168, 22 pages, 2012.

[28] L. Zhang and J. Lam, "Necessary and sufficient conditions for analysis and synthesis of Markov jump linear systems with incomplete transition descriptions," IEEE Transactions on Automatic Control, vol. 55, no. 7, pp. 1695-1701, 2010.

[29] Q. Ma, S. Xu, and Y. Zou, "Stability and synchronization for Markovian jump neural networks with partly unknown transition probabilities," Neurocomputing, vol. 74, no. 17, pp. 3404-3411, 2011.

[30] X. Luan, F. Liu, and P. Shi, "Finite-time filtering for non-linear stochastic systems with partially known transition jump rates," IET Control Theory \& Applications, vol. 4, no. 5, pp. 735-745, 2010.

[31] Y. Zhang, Y. He, M. Wu, and J. Zhang, "Stabilization for Markovian jump systems with partial information on transition probability based on free-connection weighting matrices," Automatica, vol. 47, no. 1, pp. 79-84, 2011.

[32] J. K. Hale and S. M. Verduyn Lunel, Introduction to FunctionalDifferential Equations, vol. 99 of Applied Mathematical Sciences, Springer, New York, NY, USA, 1993.

[33] X.-M. Sun, J. Zhao, and D. J. Hill, "Stability and $L_{2}$-gain analysis for switched delay systems: a delay-dependent method," Automatica, vol. 42, no. 10, pp. 1769-1774, 2006.

[34] X. Mao, "Stability of stochastic differential equations with Markovian switching," Stochastic Processes and Their Applications, vol. 79, no. 1, pp. 45-67, 1999.

[35] H. B. Ji, Algebra Foundation of Control Theory, University of Science and Technology of China Press, Hefei, China, 2008.

[36] Y. G. Sun, L. Wang, and G. Xie, "Exponential stability of switched systems with interval time-varying delay," IET Control Theory \& Applications, vol. 3, no. 8, pp. 1033-1040, 2009.

[37] J. Sun, G. P. Liu, and J. Chen, "Delay-dependent stability and stabilization of neutral time-delay systems," International Journal of Robust and Nonlinear Control, vol. 19, no. 12, pp. 13641375, 2009.

[38] P. Park, J. W. Ko, and C. Jeong, "Reciprocally convex approach to stability of systems with time-varying delays," Automatica, vol. 47, no. 1, pp. 235-238, 2011.

[39] L. Xie, "Output feedback $H_{\infty}$ control of systems with parameter uncertainty," International Journal of Control, vol. 63, no. 4, pp. 741-750, 1996.

[40] L. Xiong, J. Tian, and X. Liu, "Stability analysis for neutral Markovian jump systems with partially unknown transition 
probabilities," Journal of the Franklin Institute, vol. 349, no. 6, pp. 2193-2214, 2012.

[41] S. Mondié and V. L. Kharitonov, "Exponential estimates for retarded time-delay systems: an LMI approach," IEEE Transactions on Automatic Control, vol. 50, no. 2, pp. 268-273, 2005.

[42] P.-L. Liu, "Exponential stability for linear time-delay systems with delay dependence," Journal of the Franklin Institute, vol. 340, no. 6-7, pp. 481-488, 2003.

[43] S. Xu, J. Lam, and M. Zhong, "New exponential estimates for time-delay systems," IEEE Transactions on Automatic Control, vol. 51, no. 9, pp. 1501-1505, 2006.

[44] Y. Chen, A. Xue, R. Lu, and S. Zhou, "On robustly exponential stability of uncertain neutral systems with time-varying delays and nonlinear perturbations," Nonlinear Analysis: Theory, Methods \& Applications, vol. 68, no. 8, pp. 2464-2470, 2008.

[45] D. Wang, W. Wang, and P. Shi, "Exponential $H_{\infty}$ filtering for switched linear systems with interval time-varying delay," International Journal of Robust and Nonlinear Control, vol. 19, no. 5, pp. 532-551, 2009.

[46] D. Wang, W. Wang, P. Shi, and X.-M. Sun, "Controller failure analysis for systems with interval time-varying delay: a switched method," Circuits, Systems, and Signal Processing, vol. 28, no. 3, pp. 389-407, 2009.

[47] S. He and F. Liu, "Exponential stability for uncertain neutral systems with Markov jumps," Journal of Control Theory and Applications, vol. 7, no. 1, pp. 35-40, 2009. 


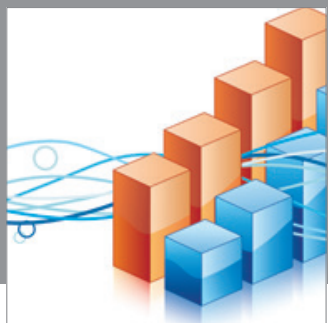

Advances in

Operations Research

mansans

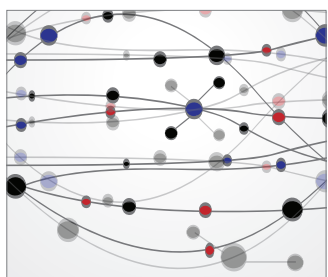

The Scientific World Journal
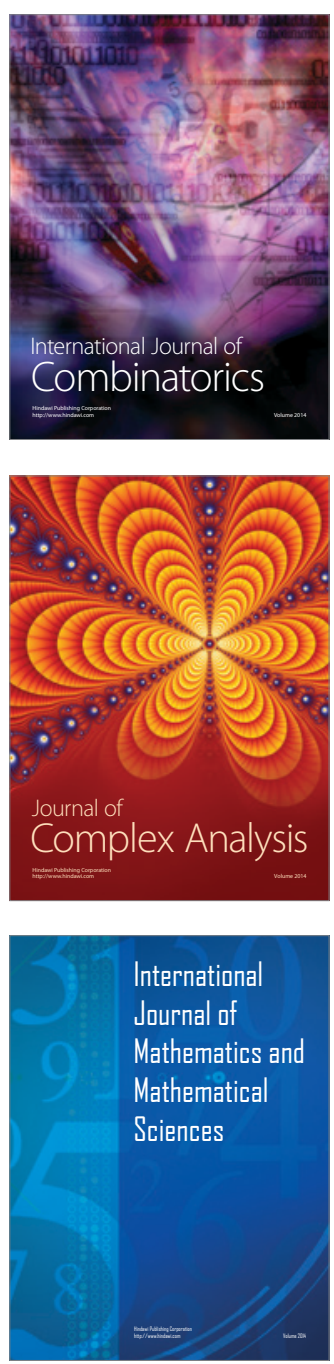
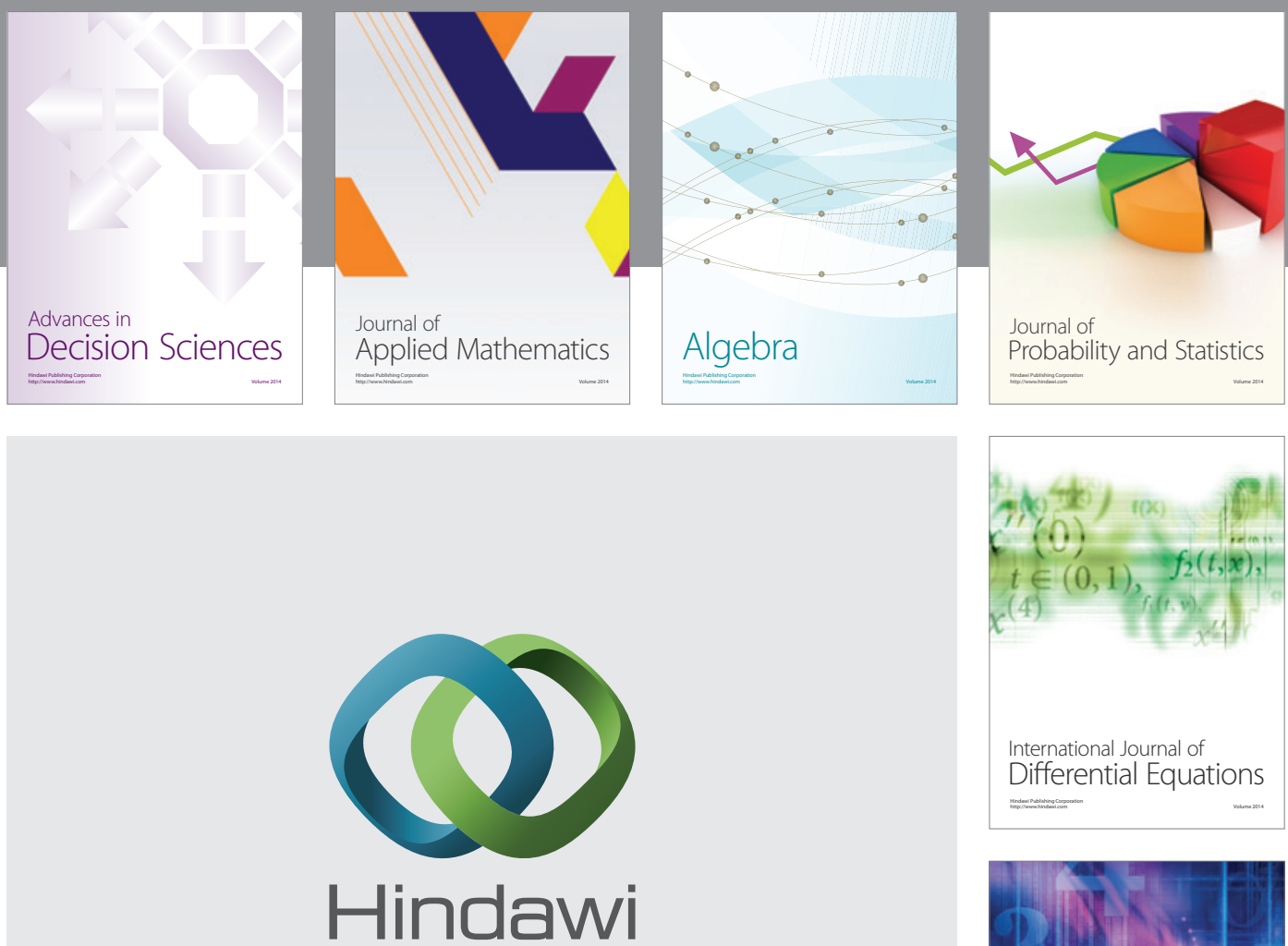

Submit your manuscripts at http://www.hindawi.com
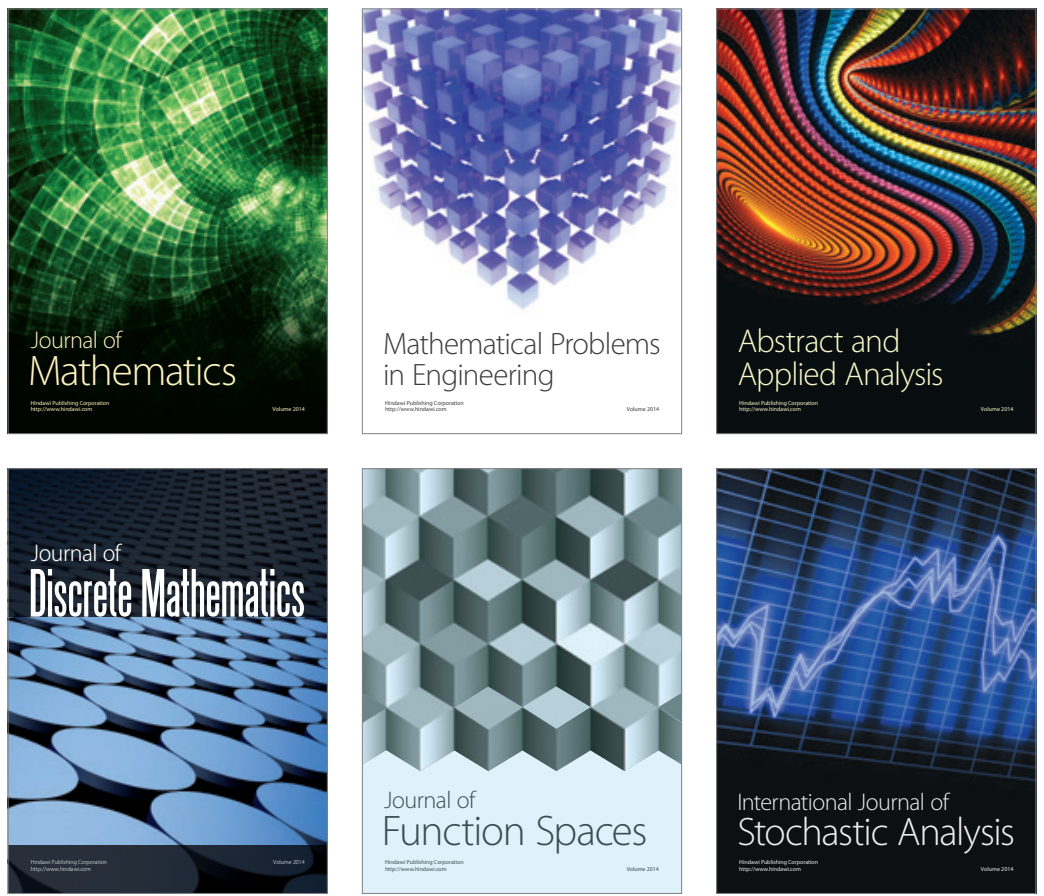

Journal of

Function Spaces

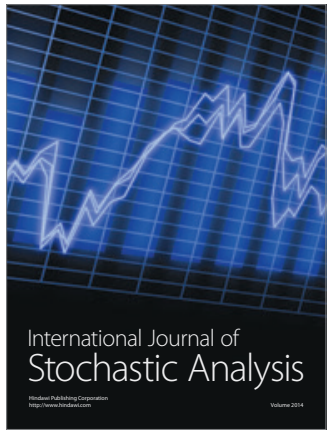

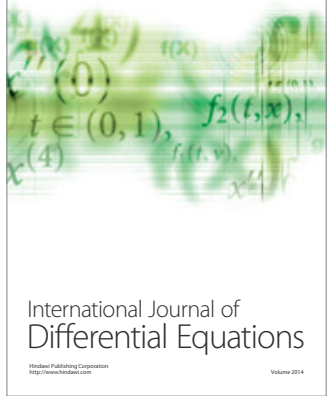
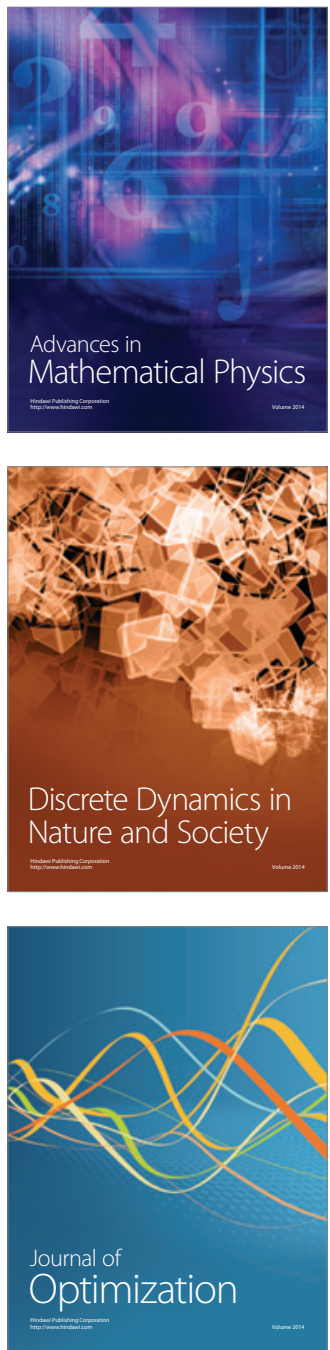